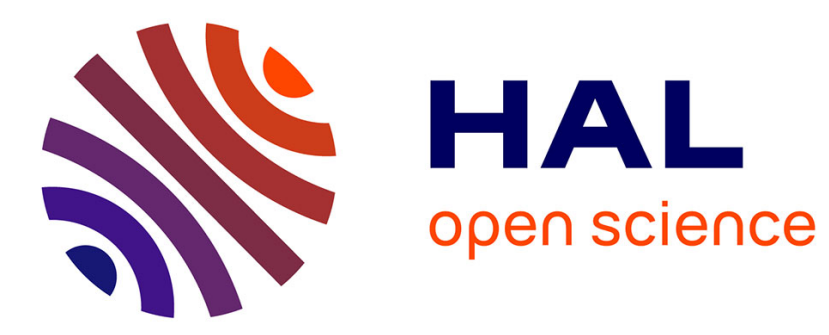

\title{
Homogenization of a neutronic critical diffusion problem with drift \\ Yves Capdeboscq
}

\section{To cite this version:}

Yves Capdeboscq. Homogenization of a neutronic critical diffusion problem with drift. Proceedings of the Royal Society of Edinburgh: Section A, Mathematics, 2002, 132 (3), pp.567-594. 10.1017/S0308210500001785 . hal-02083807

\section{HAL Id: hal-02083807 https://hal.science/hal-02083807}

Submitted on 10 Feb 2021

HAL is a multi-disciplinary open access archive for the deposit and dissemination of scientific research documents, whether they are published or not. The documents may come from teaching and research institutions in France or abroad, or from public or private research centers.
L'archive ouverte pluridisciplinaire HAL, est destinée au dépôt et à la diffusion de documents scientifiques de niveau recherche, publiés ou non, émanant des établissements d'enseignement et de recherche français ou étrangers, des laboratoires publics ou privés. 


\title{
Homogenization of a neutronic critical diffusion problem with drift
}

\author{
Yves Capdeboscq \\ Department of Mathematics, Rutgers University, 110 Frelinghuysen \\ Road, Piscataway, NJ 08854, USA (ycrc@math.rutgers.edu )
}

(MS received 27 September 2000; accepted 28 June 2001)

In this paper we study the homogenization of an eigenvalue problem for a cooperative system of weakly coupled elliptic partial differential equations, called the neutronic multigroup diffusion model, in a periodic heterogeneous domain. Such a model is used for studying the criticality of nuclear reactor cores. In a recent work in collaboration with Grégoire Allaire, it is proved that, under a symmetry assumption, the first eigenvector of the multigroup system in the periodicity cell controls the oscillatory behaviour of the solutions, whereas the global trend is asymptotically given by a homogenized diffusion eigenvalue problem. It is shown here that when this symmetry condition is not fulfilled, the asymptotic behaviour of the neutron flux, corresponding to the first eigenvector of the multigroup system, is dramatically different. This result enables to consider new types of geometrical configurations in practical nuclear reactor core computations.

\section{Introduction}

This paper is devoted to the homogenization of the following eigenvalue problem,

$$
\left.\begin{array}{rlrl}
-\epsilon^{2} \operatorname{div}\left(A_{\epsilon} \nabla \phi^{\epsilon}\right)+\Sigma_{\epsilon} \phi^{\epsilon} & =\mu^{\epsilon} \sigma \phi^{\epsilon} & & \text { in } \Omega, \\
\phi^{\epsilon} & =0 & & \text { on } \partial \Omega,
\end{array}\right\}
$$

which is, in our case, a condensed notation of the following system of $K$ equations,

$$
\left.\begin{array}{cc}
-\epsilon^{2} \operatorname{div}\left(A_{\alpha}\left(\frac{x}{\epsilon}\right) \nabla \phi_{\alpha}^{\epsilon}\right)+\sum_{\beta=1}^{K} \Sigma_{\alpha, \beta}\left(\frac{x}{\epsilon}\right) \phi_{\beta}^{\epsilon}=\mu^{\epsilon} \sum_{\beta=1}^{K} \sigma_{\alpha, \beta}\left(\frac{x}{\epsilon}\right) \phi_{\beta}^{\epsilon} & \text { in } \Omega, \\
\phi_{\alpha}^{\epsilon}=0 & \text { on } \partial \Omega,
\end{array}\right\}
$$

where the neutron flux $\phi=\left(\phi_{\alpha}\right)_{1 \leqslant \alpha \leqslant K}$ is a $K$-component vector function. In this system, each line corresponds to a balance equation for neutrons moving at a fixed speed. At each given speed or kinetic energy, $A_{\alpha}$ is the diffusion coefficient. The matrices $\Sigma$ and $\sigma$ are the total cross-section, and the fission cross-section, and the Dirichlet boundary condition implies that no neutrons enter or leave the domain. The eigenvalue $\mu$ is a measure of the balance between production and removal of neutrons in a quasi-static limit. If $\mu<1$, too many neutrons are diffused or absorbed in the core compared to their production by fission: the nuclear chain reaction dies out, and the reactor is said to be subcritical. If $\mu>1$, too many neutrons are created by fission, and the reactor is said to be supercritical. In such 
a case, absorbing media (the so-called control rods) should be added to control the reaction. Eventually, when $\mu=1$, the reactor is said to be critical: a perfect balance between fission and absorption-diffusion is obtained. We remark that (1.2) gives the spatial distribution of the neutron flux (which, in turn, yields the total power), but not its intensity, since an eigenvector is defined up to a multiplicative constant. The domain $\Omega$ is a bounded open set in $\mathbb{R}^{N}$ (the nuclear reactor core), and in this domain the coefficients are assumed to be periodic of period $\epsilon[0,1]^{N}$ (a typical fuel assembly). The small positive parameter $\epsilon$ is intended to tend to zero. The scaling of order $\epsilon^{2}$ of the diffusion parameter ensures that as the period decreases, the mean free path of a neutron must stay of the order of the periodicity cell size.

The homogenization of system (1.1) was historically studied by nuclear physicists [9]. In particular, heuristic averaging formulae had been obtained by introducing a factorizing principle, which separated local oscillations, due to the periodic heterogeneous structure of nuclear reactor cores, from a macroscopic component that took into account the finiteness of the domain. For the neutron transport model, Larsen $[15,16]$ introduced a mathematical framework in the mid 1970s. Using a formal asymptotic expansion technique, Larsen established the factorization phenomenon intuited by the physicists. Rigorous convergence results were given by Sentis [24] on a similar problem. Concerning the diffusion approximation, a rigorous justification of this factorization principle was given only recently. The one-equation (or group) model has been addressed by Allaire and Malige in [2]. When the number of equations (or groups) $K$ is greater than one, it is proved in [1] that, under a symmetry assumption (3.1), each component $\phi_{\alpha}^{\epsilon}(x)$ can be factorized in $\psi_{\alpha}(x / \epsilon)(u(x)+o(1))$, where $o(1) \rightarrow 0$ weakly in the $H^{1}$-norm. The oscillatory terms $\left(\psi_{\alpha}\right)_{1 \leqslant \alpha \leqslant K}$ are the components of the first eigenvector solution of the cell problem (1.3), which corresponds to (1.1) posed on a periodicity cell

$$
\left.\begin{array}{c}
-\operatorname{div}(A(y) \nabla \psi)+\Sigma(y) \psi=\mu^{\infty} \sigma(y) \psi \quad \text { in } Y, \\
y \rightarrow \psi(y) \quad Y \text {-periodic. }
\end{array}\right\}
$$

The macroscopical trend $u$ is the solution of the following homogenized eigenvalue problem,

$$
\begin{aligned}
-\operatorname{div}(\bar{D} \nabla u) & =\nu \bar{\sigma} u & & \text { in } \Omega, \\
u & =0 & & \text { on } \partial \Omega,
\end{aligned}
$$

where $\bar{D}$ and $\bar{\sigma}$ can be computed with the help of an auxiliary problem posed on the periodicity cell. Furthermore, equations (1.3) and (3.5) give a second-order approximation of the critical eigenvalue $\mu_{\epsilon}=\mu^{\infty}+\epsilon^{2} \nu+o\left(\epsilon^{2}\right)$. These results show, in particular, that, apart from isolating asymptotically two scales of oscillations, the homogenization procedure contracts a system of $n$ diffusion equations to the resolution of a single scalar equation giving the macroscopical trend.

The symmetry assumption (3.1) is a necessary condition for the above result to hold. It is a compatibility condition given by the Fredholm alternative, which appears not only in the convergence proof, but also in the formal derivation of the homogenized operator by a two-scale asymptotic expansion technique [18]. The geometrical disposition in new fuel assemblies (i.e. periodicity cells) is such that 


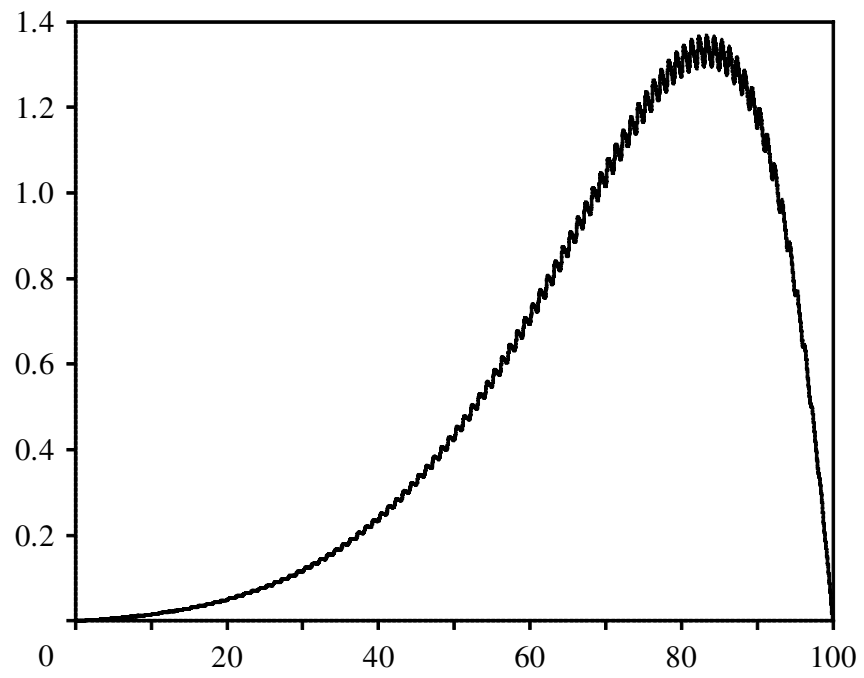

Figure 1. An example of drift $\phi_{1}^{\epsilon}$, with $\epsilon=\frac{1}{100}$ and $K=2$.

the symmetry condition is satisfied. However, the fuel depletion in the reactor core is not spatially uniform, and this symmetry property is not preserved during a fuel assembly lifetime. As a consequence, the available homogenized coefficient formulae cannot be computed. In this paper, the case when the symmetry hypothesis (3.1) is not satisfied is addressed. Most of the paper is dedicated to obtaining the exact homogenized coefficients, which require the introduction of $\theta$-exponential periodic eigenvalue problems. We then show that, for a small perturbation of the symmetry condition (3.1), approximate formulae for the homogenized coefficients can be obtained with the computation of an auxiliary problem simply posed on the periodicity cell, as it is the case when the symmetry condition is fulfilled.

Figure 1 represents a situation where the symmetry condition (3.1) is not satisfied. The computation was made for a two-group model $(K=2)$, in one dimension of space $(N=1)$, with a periodicity cell composed of the juxtaposition of three different materials. The first component of the flux $\phi_{1}^{\epsilon}$ is represented for $\epsilon=0.01$. The second component $\phi_{2}^{\epsilon}$ is very similar.

In such a case, the microscopic scale does not only control the oscillatory behaviour of the eigenfunctions, but also induces an exponential drift. One then needs to introduce the following family of $\theta$-exponential periodic problems,

$$
\left.\begin{array}{c}
-\operatorname{div}\left(A(y) \nabla \psi_{\theta}\right)+\Sigma(y) \psi_{\theta}=\lambda(\theta) \sigma(y) \psi_{\theta}, \\
y \rightarrow \psi_{\theta}(y) \mathrm{e}^{-\theta \cdot y} \quad Y \text {-periodic, }
\end{array}\right\}
$$

where $\theta \in \mathbb{R}^{N}$ is a parameter. The function $\theta \rightarrow \lambda(\theta)$ is a one-bump function with a maximum $\lambda_{\infty}$ attained for a unique $\theta=\theta_{\infty}$. The main result of this paper is theorem 3.3, which shows that each component $\phi_{\alpha}^{\epsilon}$ of the solution of (1.1) can be factorized in $\psi_{\infty, \alpha}(x / \epsilon)(u(x)+o(1))$, where $o(1) \rightarrow 0$ weakly in the $H^{1}$-norm, $(\nu, u)$ is a solution of an homogenized eigenvalue problem similar to $(3.5)$, and the $\epsilon$ dependent terms $\left(\psi_{\infty, \alpha}\right)_{1 \leqslant \alpha \leqslant K}$ are the components of the first eigenvector solution 
of the $\theta_{\infty}$-exponential cell problem (1.4), i.e.

$$
\psi_{\infty, \alpha}\left(\frac{x}{\epsilon}\right)=\exp \left(\frac{\theta_{\infty} \cdot x}{\epsilon}\right) \varphi_{\infty}\left(\frac{x}{\epsilon}\right),
$$

where $\varphi_{\infty}$ is a $Y$-periodic function. A similar result on a model problem is exposed in [7].

The paper is organized as follows. In $\S 2$ we give detailed assumptions on the coefficients and we recall existence and regularity results for systems (1.2) and (1.4). We also state the existence of a maximum for $\theta \rightarrow \lambda(\theta)$. In $\S 3$ we give a precise statement of the homogenization theorem 3.3 and its proof upon admitting the results stated in $\S 2$. Section 4 is devoted to the proof of the existence of a maximum drift eigenvalue. Finally, in $\S 5$, it is shown that, in the case of a small drift, minor modification needs to be brought to the computational scheme of the drift-free situation to obtain a second-order approximation of the maximum eigenvalue $\lambda_{\infty}$ or a first-order evaluation of the drift factor $\theta_{\infty}$, and an approximate formula for the homogenized diffusion matrix is proposed.

\section{Existence and regularity results}

Let us now detail the hypothesis and notation that will be used throughout this paper. Recall that $N$ is the space dimension, and $K$ is the number of equations (corresponding to different energy groups). We adopt the convention that Latin indices $i, j$ belong to $\{1, \ldots, N\}$, i.e. refer to spatial coordinates, while Greek indices $\alpha, \beta$ vary in $\{1, \ldots, K\}$, i.e. refer to the group label. It is essential to note in (1.2) that the system is weakly coupled, i.e. there appear no derivatives in the coupling terms. The operator $-\operatorname{div}(A(x / \epsilon) \nabla \phi)$, where $\phi=\left(\phi_{1}, \ldots, \phi_{K}\right)$ will always stand for

$$
-\left[\begin{array}{ccc}
\operatorname{div}\left(A_{1}(x / \epsilon) \nabla(\cdot)\right) & 0 & 0 \\
0 & \operatorname{div}\left(A_{\alpha}(x / \epsilon) \nabla(\cdot)\right) & 0 \\
0 & 0 & \operatorname{div}\left(A_{K}(x / \epsilon) \nabla(\cdot)\right)
\end{array}\right]\left[\begin{array}{c}
\phi_{1} \\
\vdots \\
\phi_{\alpha} \\
\vdots \\
\phi_{K}
\end{array}\right]
$$

and each $\left(A_{\alpha}\right)_{1 \leqslant \alpha \leqslant K}$ is a symmetric $N \times N$ matrix. The small parameter $\epsilon$ is intended to tend to zero. Our second assumption is that all coefficients in (1.2) are measurable and bounded, i.e. $A_{\alpha, i j}(y), \Sigma_{\alpha, \beta}(y), \sigma_{\alpha, \beta}(y) \in L^{\infty}(Y)$ for $1 \leqslant i$, $j \leqslant N$ and $1 \leqslant \alpha, \beta \leqslant K$. This is the natural functional framework, since we want to model heterogeneous media having discontinuous properties. However, for a technical reason, i.e. theorem 2.5 below, we shall also suppose, for $1 \leqslant i, j \leqslant N$ and $1 \leqslant \alpha \leqslant K$,

$$
A_{\alpha, i j}(y) \in C^{1}(Y) .
$$

The diffusion matrices are assumed to be coercive, i.e. there exists a positive constant $C>0$ such that, for any $\alpha \in\{1, \ldots, K\}$ and for any $\xi \in \mathbb{R}^{N}$,

$$
A_{\alpha}(y) \xi \cdot \xi \geqslant C|\xi|^{2} \quad \text { for a.e. } y \in Y \text {. }
$$


For physical reasons, all fission cross-sections are non-negative (fission is a production process), and the matrix $\sigma$ is non-trivial,

$$
\sigma \geqslant 0, \quad \sigma \not \equiv 0
$$

This is the minimal assumption that can be made on the fission matrix, which enables our results to cover all physical multigroup diffusion models. The proof of theorem 2.8 can be simplified when $\sigma$ is supposed diagonal or strictly positive. The matrix $\Sigma$ of the total (or scattering) cross-sections is diagonal dominant, and we suppose that there is a net absorption in each group,

$$
\Sigma_{\alpha, \alpha} \geqslant C>0, \quad \Sigma_{\alpha, \beta} \leqslant 0 \quad \text { if } \alpha \neq \beta \quad \text { and } \quad \sum_{\beta=1}^{K} \Sigma_{\alpha, \beta} \geqslant 0, \quad 1 \leqslant \alpha \leqslant K .
$$

We also suppose that the system is fully coupled and essentially positive (and therefore cooperative in the sense of [26]), for example,

$$
-\Sigma_{\alpha, \alpha-1} \geqslant C>0
$$

Furthermore, to ensure that the periodic eigenvalue $\mu^{\infty}$ is not zero, we make the following hypothesis:

$$
\exists \alpha_{0} \text { such that } \sum_{\beta=1}^{K} \Sigma_{\alpha_{0}, \beta} \not \equiv 0 .
$$

The fact that the system is cooperative is very important in our analysis. For such systems, positivity properties of both matrix equations and elliptic differential operators support each other. Positivity of the solutions follows from the classical maximum principle for elliptic equations. This is not the case for non-cooperative systems.

Finally, our last assumption is that the nuclear reactor core is periodic, i.e. all coefficients $A(y), \Sigma(y)$ and $\sigma(y)$ are $Y$-periodic functions. This hypothesis is crucial for the homogenization procedure. In particular, our results do not hold true any longer if the coefficients are the product of periodic functions with macroscopic modulations, as, for example, $\Sigma(x, x / \epsilon)$, with a $Y$-periodic function $\Sigma(x, y)$.

REMARK 2.1. Assumption (2.1) is used in the proof of theorem 2.5. It is clearly a restriction to the general case, and is probably not compulsory for the establishment of this existence result.

Let us now turn to the existence of solutions for the eigenvalue problems (1.2), $(1.3)$.

TheOREm 2.2. Under assumptions (2.2) and (2.3)-(2.6), problem (1.2) admits at least one, and at most a countable number of, eigenvalues (possibly complex), with associated eigenvectors in $H_{0}^{1}(\Omega)^{K}$. Furthermore, the first eigenvalue of (1.2) (i.e. the smallest in modulus) is real and simple, and its corresponding eigenvector can be chosen to be positive in $\Omega$ (i.e. each component is positive). 
REMARK 2.3. Throughout the paper, we label the eigenvalues by increasing order of their modulus. We normalize the eigenvectors of (1.2) so that their norm is equal to one,

$$
\sum_{\alpha=1}^{K} \int_{\Omega} \phi_{\alpha}^{\epsilon}(x) \cdot \phi_{\alpha}^{\epsilon}(x) \mathrm{d} x=1 .
$$

For all the cell problems (1.3), (1.4), (2.7) and (2.8), we adopt the following normalization:

$$
\sum_{\alpha=1}^{K} \int_{Y} \psi_{\alpha}(y) \cdot \psi_{\alpha}(y) \mathrm{d} y=1 \quad \text { and } \quad \sum_{\alpha, \beta=1}^{K} \int_{Y} \sigma_{\alpha, \beta}(y) \psi_{\beta}(y) \cdot \psi_{\alpha}^{*}(y) \mathrm{d} y=1 .
$$

Theorem 2.2 was first proved by Habetler and Martino [11], with the help of Green function inequalities given by Stampacchia and of the Krein-Rutman theorem (see $[3,14,25])$. A modern exposition of this result for neutronic models may be found in the book of Planchard [22]. In the general context of weakly coupled cooperative elliptic systems, this result has independently been proved by Mitidieri and Sweers [19].

In order to compute the homogenized coefficients, when no drift appears, one also need to introduce the adjoint cell problem of (1.3),

$$
\left.\begin{array}{c}
-\operatorname{div}\left(A(y) \nabla \psi^{*}\right)+\Sigma^{*}(y) \psi^{*}=\mu^{\infty} \sigma^{*}(y) \psi^{*}, \\
y \rightarrow \psi^{*}(y) \quad Y \text {-periodic, }
\end{array}\right\}
$$

where $\Sigma^{*}$ and $\sigma^{*}$ are the adjoint or transposed matrices of $\Sigma$ and $\sigma$, respectively, and $\mu^{\infty}$ is the first eigenvalue (the same as for (1.3)).

In a similar manner, when there is a drift phenomenon, the homogenized coefficients will involve the adjoint eigenvalue problem of (1.4), relatively to the scalar product in $L^{2}(Y)^{K}$,

$$
\left.\begin{array}{c}
-\operatorname{div}\left(A(y) \nabla \psi_{\theta}^{*}\right)+\Sigma^{*}(y) \psi_{\theta}^{*}=\lambda(\theta) \sigma^{*}(y) \psi_{\theta}^{*}, \\
y \rightarrow \psi_{\theta}^{*}(y) \mathrm{e}^{\theta \cdot y} \quad Y \text {-periodic. }
\end{array}\right\}
$$

Because of assumptions (2.4) and (2.6), there exists a periodic supersolution for system (1.3). Thus the existence of a first eigenvalue for the cell problem (1.3) is a corollary of theorem 2.2 (see [26]).

COROllary 2.4. Under assumptions (2.2) and (2.3)-(2.6), problem (1.3) admits at least one, and at most a countable number of, eigenvalues with associated eigenvectors in $H_{\#}^{1}(Y)^{K}$. Furthermore, problem (1.3) and its adjoint have a common first eigenvalue $\mu^{\infty}$ that is real and simple, and its corresponding eigenvectors $\psi$ and $\psi^{*}$ can be chosen to be positive in $Y$.

We recall that the space $H_{\#}^{1}(Y)$ of periodic functions is defined by

$$
H_{\#}^{1}(Y)=\left\{\varphi \in H^{1}(Y) \text { such that } y \rightarrow \varphi(y) \text { is } Y \text {-periodic }\right\} .
$$

The existence of a first eigenpair for the eigenvalue problem (1.4) is less classical. Throughout this paper we denote by $\left(\psi_{\theta, \alpha}\right)_{1 \leqslant \alpha \leqslant K}$ (respectively, $\left.\left(\psi_{\theta, \alpha}^{*}\right)_{1 \leqslant \alpha \leqslant K}\right)$ the 
components of the eigenvector $\psi_{\theta}$ of (1.4) (respectively, $\psi_{\theta}^{*}$ of $(2.7)$ ) associated to the first eigenvalue $\lambda(\theta)$.

In the particular case when $\sigma$ is the identity matrix in $\mathbb{R}^{K \times K}$, noted $I_{K}$, we shall denote the first eigenvalue $\Lambda(\theta)$, and the corresponding direct and adjoint eigenvectors $\vartheta_{\theta}$ and $\vartheta_{\theta}^{*}$. Let us first address this particular case.

TheOREm 2.5. Suppose that $\sigma=I_{K}$. Under assumptions (2.2), (2.4)-(2.6) and (2.1), for all $\theta \in \mathbb{R}^{N}$, there is a unique normalized strictly positive eigenfunction $\vartheta_{\theta} \in\left(W_{\mathrm{loc}}^{2, N}(Y) \cap C(\bar{Y})\right)^{K}$ to (1.4) and $\vartheta_{\theta}^{*} \in\left(W_{\mathrm{loc}}^{2, N}(Y) \cap C(\bar{Y})\right)^{K}$ to (2.8). The first eigenvalue $\Lambda(\theta)$ of (1.4) and (2.8) is the same. It is of geometric and algebraic multiplicity equal to one.

Furthermore, the application $\theta \rightarrow \Lambda(\theta)$ is in $C^{\infty}\left(\mathbb{R}^{N}, \mathbb{R}\right)$.

Proof. In order to work in the periodic function space $H_{\#}^{1}(Y)^{K}$, we introduce the following family of operators, variationally defined by $\forall 1 \leqslant \alpha \leqslant K, \forall \phi, \psi \in H_{\#}^{1}(Y)$,

$$
\left\langle A_{\alpha}(\theta) \phi, \psi\right\rangle=\sum_{i, j=1}^{N} \int_{Y} A_{\alpha, i, j}(y)\left(\frac{\partial \phi}{\partial y_{j}}-\theta_{j} \phi\right) \cdot\left(\frac{\partial \psi}{\partial y_{i}}+\theta_{i} \psi\right) \mathrm{d} y,
$$

which can be written, because of assumption (2.1), under the following classical form, $\forall 1 \leqslant \alpha \leqslant K$,

$$
\forall \phi \in H_{\#}^{1}(Y), \quad A_{\alpha}(\theta)(\phi)=-\operatorname{div}\left(A_{\alpha} \nabla \phi\right)+\theta \cdot A_{\alpha} \nabla \phi+\left(\operatorname{div}\left(A_{\alpha} \theta\right)-A_{\alpha} \theta \cdot \theta\right) \phi .
$$

With the change of variable $\vartheta_{\theta}=\mathrm{e}^{\theta \cdot y} \varphi(y)$, we obtain that system (1.4) defining $\vartheta_{\theta}$ writes under the following equivalent form

$$
\left.\begin{array}{rl}
A(\theta) \varphi_{\theta} & +\left(\Sigma+c I_{K}\right) \varphi_{\theta}=(\Lambda(\theta)+c) \varphi_{\theta}, \\
y & \rightarrow \varphi_{\theta}(y) \quad Y \text {-periodic. }
\end{array}\right\}
$$

For any constant $c \in \mathbb{R}$, choose

$$
c=\max _{1 \leqslant \alpha \leqslant K} \sup _{y \in[0,1]}\left(\left|\operatorname{div}\left(A_{\alpha} \theta\right)\right|+\left|A_{\alpha}(y) \theta \cdot \theta\right|\right),
$$

and theorem 2.6 (proved in [26]) allows us to conclude that there exists a unique positive eigenpair $\left(\varphi_{\theta}, \Lambda(\theta)+c\right)$ solution of $(2.10)$, with $\varphi_{\theta} \in\left(W_{\operatorname{loc}}^{2, N}(Y) \cap C(\bar{Y})\right)^{K}$ and $\Lambda(\theta)+c>0$ (unique after normalizing). The same argument holds for the adjoint equation of (2.10). The fact that the first eigenvalue of the direct and adjoint problem is the same, and that it is simple and isolated, are consequences of the Krein-Rutman theorem. Because of its simplicity, and of the fact that $A_{\theta}$ in (2.9) depends polynomially on $\theta$, the regularity of $\theta \rightarrow \Lambda(\theta)$ is a consequence of Kato-Rellich theorem (see, for example, $[12,23]$ ).

THEOREM 2.6 (cf. [26]). Under assumptions (2.2), (2.4) and (2.5), if there exists a positive supersolution of (2.10), then there is a unique strictly positive eigenfunction $\varphi_{\theta} \in\left(W_{\mathrm{loc}}^{2, N}(Y) \cap C(\bar{Y})\right)^{K}$ solution of (2.10), for some $\Lambda(\theta)>0$ (unique after normalizing).

Define the open connected subset

$$
U=\Lambda^{-1}\left(\mathbb{R}_{+}^{*}\right) \subset \mathbb{R}^{N}
$$


It is non-empty, since $0 \in U$ by corollary 2.4. The connectedness of the set $U$ will be shown in lemma 4.3. Then the following result, due to Mitidieri and Sweers [19], proves the existence of solutions for systems (1.4) and (2.8) for any $\sigma$ satisfying (2.3).

Proposition 2.7 (cf. [19]). Let $U$ be the open subset of $\mathbb{R}^{N}$ defined by (2.11). Under assumptions (2.2), (2.3)-(2.6) and (2.1), for all $\theta \in U$, there is a unique normalized strictly positive eigenfunction $\psi_{\theta} \in\left(W_{\mathrm{loc}}^{2, N}(Y) \cap C(\bar{Y})\right)^{K}$ to (1.4) and $\psi_{\theta}^{*} \in\left(W_{\mathrm{loc}}^{2, N}(Y) \cap C(\bar{Y})\right)^{K}$ to (2.8). The first eigenvalue $\lambda(\theta)$ of (1.4) and (2.8) is the same. It is of geometric and algebraic multiplicity equal to one.

The introduction of the $\theta$-exponential cell problems will be proved useful in obtaining the homogenized limit of system (1.2). In particular, the following result will be the key ingredient that will enable the generalization of the results obtained in [1] to the general situation.

THEOREM 2.8. Let $\lambda(\theta)$ be the first eigenvalue of system (1.4), and $U$ be given by (2.11). The application $\theta \rightarrow \lambda(\theta)$ is in $C^{\infty}\left(U, \mathbb{R}_{+}^{*}\right)$ and admits a maximum $\lambda_{\infty}$, which is obtained for a unique $\theta=\theta_{\infty}$. It is characterized by the following relation:

$$
\sum_{\alpha=1}^{K} \int_{Y} A_{\alpha}(y)\left(\psi_{\infty, \alpha}(y) \nabla \psi_{\infty, \alpha}^{*}(y)-\psi_{\infty, \alpha}^{*}(y) \nabla \psi_{\infty, \alpha}(y)\right)=0 .
$$

Section 4 is devoted to the proof of this result.

\section{Main results}

Let us first recall the result obtained in the case when the following symmetry condition is satisfied,

$$
\sum_{\alpha=1}^{K} \int_{Y} A_{\alpha}(y)\left(\psi_{\alpha} \nabla \psi_{\alpha}^{*}-\psi_{\alpha}^{*} \nabla \psi_{\alpha}\right) \mathrm{d} y=0,
$$

where $\left(\psi_{\alpha}\right)_{1 \leqslant \alpha \leqslant K}$ (respectively, $\left(\psi_{\alpha}^{*}\right)_{1 \leqslant \alpha \leqslant K}$ ) are the components of the first eigenvector $\psi$ of (1.3) (respectively, $\psi^{*}$ of $(2.7)$ ).

The homogenized coefficients $\bar{\sigma}$ and $\bar{D}$ are then defined in the following way,

$$
\bar{\sigma}=\sum_{\alpha, \beta=1}^{K} \int_{Y} \sigma_{\alpha, \beta}(y) \psi_{\beta}(y) \psi_{\alpha}^{*}(y) \mathrm{d} y,
$$

and $\bar{D}$ is a $N \times N$ matrix defined by its entries

$$
\begin{aligned}
\bar{D}_{i, j}=\sum_{\alpha=1}^{K} \int_{Y} & A_{\alpha} \psi_{\alpha} \psi_{\alpha}^{*} \nabla\left(y_{i}+\xi_{i, \alpha}\right) \nabla\left(y_{j}+\xi_{j, \alpha}\right) \mathrm{d} y \\
& \quad+\sum_{\alpha, \beta=1}^{K} \int_{Y} \frac{1}{2} \psi_{\alpha}^{*} \psi_{\beta}\left(\mu^{\infty} \sigma_{\alpha, \beta}-\Sigma_{\alpha, \beta}\right)\left(\xi_{i, \alpha}-\xi_{i, \beta}\right)\left(\xi_{j, \alpha}-\xi_{j, \beta}\right) \mathrm{d} y,
\end{aligned}
$$

where, for each $1 \leqslant i \leqslant N$, the components $\left(\xi_{i, \alpha}\right)_{1 \leqslant \alpha \leqslant K}$ are defined by

$$
\xi_{i, \alpha}=\frac{\zeta_{i, \alpha}}{\psi_{\alpha}}
$$


and $\zeta_{i}=\left(\zeta_{i, \alpha}\right)_{1 \leqslant \alpha \leqslant K}$ is the solution of

$$
\left.\begin{array}{c}
-\operatorname{div}\left(A(y) \nabla \zeta_{i}\right)+\Sigma(y) \zeta_{i}=\mu^{\infty} \sigma(y) \zeta_{i}+Z_{i} \text { in } Y, \\
y \rightarrow \zeta_{i}(y) \quad Y \text {-periodic, }
\end{array}\right\}
$$

where the right-hand side $Z_{i}$ has components

$$
Z_{i, \alpha}=\frac{1}{\psi_{\alpha}(y)} \operatorname{div}\left(A_{\alpha}(y) \psi_{\alpha}^{2}(y) \nabla y_{i}\right) \quad \text { for } 1 \leqslant \alpha \leqslant K .
$$

REMARK 3.1. Equation (3.4) is of the same type as the cell eigenvalue problem (1.3), but with a source term. Therefore, it admits a solution provided that the Fredholm alternative holds, i.e. the source term must be orthogonal to the adjoint first eigenvector $\psi^{*}$. This is precisely the symmetry condition (3.1).

Theorem 3.2 (cf. [1]). Assume that the symmetry condition (3.1) is satisfied. Let $\left(\psi_{\alpha}\right)_{1 \leqslant \alpha \leqslant K}$ (respectively, $\left(\psi_{\alpha}^{*}\right)_{1 \leqslant \alpha \leqslant K}$ ) be the components of the eigenvector $\psi$ of (1.3) (respectively, $\psi^{*}$ of (2.7)) associated to the first eigenvalue $\mu^{\infty}$. Let $\left(\phi^{\epsilon, m}, \lambda^{\epsilon, m}\right)$ be the mth eigenpair of system (1.2). Then

$$
\begin{aligned}
& \phi_{\alpha}^{\epsilon, m}=u_{\alpha}^{\epsilon, m}(x) \psi_{\alpha}\left(\frac{x}{\epsilon}\right) \quad \forall \alpha \in\{1, \ldots, K\}, \\
& \lambda^{\epsilon, m}=\mu^{\infty}+\epsilon^{2} \nu^{m}+o\left(\epsilon^{2}\right),
\end{aligned}
$$

where, up to a subsequence, each component $u_{\alpha}^{\epsilon, m}$ converges weakly in $H_{0}^{1}(\Omega)$ to the same limit $u^{m}$, which is an eigenvector associated to the mth eigenvalue $\nu^{m}$ of the scalar homogenized problem

$$
\left.\begin{array}{rlrl}
-\operatorname{div}(\bar{D} \nabla u(x)) & =\nu \bar{\sigma} u(x) & & \text { in } \Omega, \\
u & =0 & & \text { on } \partial \Omega .
\end{array}\right\}
$$

The homogenized coefficient $\bar{\sigma}$ is given by (3.2). The homogenized $N \times N$ matrix $\bar{D}$ given by (3.3) is positive definite.

When (3.1) is not satisfied, equation (3.4) does not admit a solution, and consequently the homogenized matrix $\bar{D}$ is not well defined. Let $\left(\psi_{\infty}, \psi_{\infty}^{*}, \lambda_{\infty}\right)$ be the normalized direct and adjoint eigenvector and eigenvalue of (1.4) and (2.8), where $\theta=\theta_{\infty}$ is the maximum defined in theorem 2.8. The homogenized coefficients $\bar{D}_{\infty}$ and $\bar{\sigma}_{\infty}$ are then defined in the following way,

$$
\bar{\sigma}_{\infty}=\sum_{\alpha, \beta=1}^{K} \int_{Y} \sigma_{\alpha, \beta}(y) \psi_{\infty, \beta}(y) \psi_{\infty, \alpha}^{*}(y) \mathrm{d} y
$$

and the $N \times N$ matrix $\bar{D}_{\infty}$ is defined by its entries

$$
\begin{aligned}
\bar{D}_{\infty, i, j}=\sum_{\alpha=1}^{K} \int_{Y} A_{\alpha} \psi_{\infty, \alpha} \psi_{\infty, \alpha}^{*} \nabla\left(y_{i}+\xi_{i, \alpha}^{\infty}\right) \nabla\left(y_{j}+\xi_{j, \alpha}^{\infty}\right) \mathrm{d} y \\
\quad+\sum_{\alpha, \beta=1}^{K} \int_{Y} \frac{1}{2} \psi_{\infty, \alpha}^{*} \psi_{\infty, \beta}\left(\lambda_{\infty} \sigma_{\alpha, \beta}-\Sigma_{\alpha, \beta}\right)\left(\xi_{i, \alpha}^{\infty}-\xi_{i, \beta}^{\infty}\right)\left(\xi_{j, \alpha}^{\infty}-\xi_{j, \beta}^{\infty}\right) \mathrm{d} y,
\end{aligned}
$$


where, for each $1 \leqslant i \leqslant N$, the components $\left(\xi_{i, \alpha}^{\infty}\right)_{1 \leqslant \alpha \leqslant K}$ are defined by

$$
\xi_{i, \alpha}^{\infty}=\frac{\zeta_{i, \alpha}^{\infty}}{\psi_{\infty, \alpha}}
$$

and $\zeta_{i}^{\infty}=\left(\zeta_{i, \alpha}^{\infty}\right)_{1 \leqslant \alpha \leqslant K}$ is the solution of

$$
\left.\begin{array}{c}
-\operatorname{div}\left(A(y) \nabla \zeta_{i}^{\infty}\right)+\Sigma(y) \zeta_{i}^{\infty}=\lambda_{\infty} \sigma(y) \zeta_{i}^{\infty}+Z_{i}^{\infty} \text { in } Y, \\
y \rightarrow \zeta_{i}^{\infty}(y) \exp \left(-\theta_{\infty} \cdot y\right) \quad Y \text {-periodic, }
\end{array}\right\}
$$

where the right-hand side $Z_{i}^{\infty}$ has components

$$
Z_{i, \alpha}^{\infty}=\frac{1}{\psi_{\infty, \alpha}(y)} \operatorname{div}\left(A_{\alpha}(y) \psi_{\infty, \alpha}^{2}(y) \nabla y_{i}\right) \quad \text { for } 1 \leqslant \alpha \leqslant K .
$$

The existence of a solution for (3.8) will be given by lemma 3.8 .

Let us now state our main result, when (3.1) is not satisfied.

TheOREm 3.3. Let $\left(\phi^{\epsilon, m}, \lambda^{\epsilon, m}\right)$ be the mth eigenpair of (1.2). Let $\left(\psi_{\infty}, \psi_{\infty}^{*}, \lambda_{\infty}\right)$ be the normalized direct and adjoint eigenvector and eigenvalue of (1.4) and (2.8), where $\theta=\theta_{\infty}$ is the maximum defined in theorem 2.8. Then

$$
\begin{aligned}
\phi_{\alpha}^{\epsilon, m} & =u_{\alpha}^{\epsilon, m}(x) \psi_{\infty, \alpha}\left(\frac{x}{\epsilon}\right) \quad \forall \alpha \in\{1, \ldots, K\}, \\
\lambda^{\epsilon, m} & =\lambda_{\infty}+\epsilon^{2} \nu^{m}+o\left(\epsilon^{2}\right),
\end{aligned}
$$

where, up to a subsequence, each component $u_{\alpha}^{\epsilon, m}$ converges weakly in $H_{0}^{1}(\Omega)$ to the same limit $u^{m}$, which is an eigenvector associated to the mth eigenvalue $\nu^{m}$ of the scalar homogenized problem

$$
\left.\begin{array}{rlrl}
-\operatorname{div}\left(\bar{D}_{\infty} \nabla u(x)\right) & =\nu \bar{\sigma}_{\infty} u(x) & & \text { in } \Omega, \\
u & =0 & & \text { on } \partial \Omega .
\end{array}\right\}
$$

The homogenized coefficient $\bar{\sigma}_{\infty}$ is given by (3.6). The homogenized $N \times N$ matrix $\bar{D}_{\infty}$ given by (3.7) is positive definite.

REMARK 3.4. According to equation (2.12), the maximum eigenvalue $\lambda_{\infty}$ is equal to $\mu^{\infty}$ if and only if the symmetry condition (3.1) is satisfied. Therefore, theorem 3.2 appears to be a particular case of theorem 3.3 .

REMARK 3.5. If the wording of theorems 3.2 and 3.3 are very similar, the conclusion they lead to on the behaviour of the eigenvectors of system (1.2) are very different. Indeed, each of the components $\phi_{\alpha}^{\epsilon}$ of $\phi^{\epsilon}$ is asymptotically of the following form,

$$
\exp \left(\theta_{\infty} \cdot \frac{x}{\epsilon}\right) \varphi_{\alpha}\left(\frac{x}{\epsilon}\right) u(x) \quad \text { a.e. } x \in \Omega,
$$

where $\varphi_{\alpha}$ is a $Y$-periodic function. Thus, when $\theta_{\infty} \neq 0$, i.e. when the symmetry condition (3.1) is not satisfied, $\phi^{\epsilon}$ tends to vanish inside $\Omega$, and an exponential drift of order $1 / \epsilon$ and of direction $\theta_{\infty}$ concentrates each of the component of the $\phi^{\epsilon}$ towards the boundary of the domain. Such a phenomenon does not appear in the 
case of a self-adjoint model such as the scalar (one energy group) neutronic diffusion model. A similar result on a model problem is exposed in [7]. This exponential drift behaviour has already been treated in $[6,13,20,21]$ for comparable parabolic problems. For the neutron transport model, this drift phenomenon is treated in [5].

The following sequence of intermediate results are the elements needed for the completion of theorem 3.3.

For a fixed $\theta \in U$, where $U$ is the open subset of $\mathbb{R}^{N}$ defined by (2.11) and given in proposition 2.7 as the domain of definition of the $\theta$-exponential problem (1.4), let us follow the steps of the proof of theorem 3.2 proved in [1]. Propositions 3.6, 3.10 and lemmas 3.7 and 3.8 correspond to propositions $3.7,3.10$ and lemmas 3.9 and 5.2 in [1], where they are written for $\theta=0$. Because of the regularity properties of $\psi_{\theta}$ and $\psi_{\theta}^{*}$ recalled in proposition 2.7 , the proofs can be extended without difficulty to any $\theta \in U$.

Proposition 3.6. Let $\theta \in U$, with $U$ given by (2.11). The multigroup eigenvalue problem (1.2) is equivalent to the following eigenvalue problem,

$$
\left.\begin{array}{c}
-\operatorname{div}\left(D_{\theta}\left(\frac{x}{\epsilon}\right) \nabla u^{\epsilon}\right)+\frac{1}{\epsilon^{2}} Q_{\theta}^{\epsilon}\left(u^{\epsilon}\right)=\nu^{\epsilon} B_{\theta}\left(\frac{x}{\epsilon}\right) u^{\epsilon} \quad \text { in } \Omega, \\
u^{\epsilon} \in H_{0}^{1}(\Omega)^{K},
\end{array}\right\}
$$

where the components $\left(u_{\alpha}^{\epsilon}\right)_{1 \leqslant \alpha \leqslant K}$ of $u^{\epsilon}$ are defined by

$$
u_{\alpha}^{\epsilon}(x)=\frac{\phi_{\alpha}^{\epsilon}(x)}{\psi_{\theta, \alpha}(x / \epsilon)},
$$

the eigenvalue $\nu^{\epsilon}$ is defined by

$$
\nu^{\epsilon}=\frac{\mu^{\epsilon}-\lambda(\theta)}{\epsilon^{2}}
$$

$D(y)$ is a $Y$-periodic fourth-order tensor that is block diagonal, i.e.

$$
D=\operatorname{diag}\left(D_{\theta, 1}, \ldots, D_{\theta, K}\right),
$$

with

$$
D_{\theta, \alpha}(y)=\psi_{\theta, \alpha}(y) \psi_{\theta, \alpha}^{*}(y) A_{\alpha}(y) \quad \forall \alpha \in 1, \ldots, K,
$$

$B$ is a $K \times K Y$-periodic matrix with entries

$$
\left(B_{\theta}\right)_{\alpha, \beta}(y)=\sigma_{\alpha, \beta}(y) \psi_{\theta, \beta}(y) \psi_{\theta, \alpha}^{*}(y),
$$

and $Q^{\epsilon}$ is a continuous linear operator from $H_{0}^{1}(\Omega)^{K}$ into $H^{-1}(\Omega)^{K}$, defined by (3.15). Furthermore, there exist two positive constants $C>c>0$ (independent of $\epsilon)$ such that, for any $u \in H_{0}^{1}(\Omega)^{K}$,

$$
C \sum_{\alpha, \beta=1}^{K}\left\|u_{\alpha}-u_{\beta}\right\|_{L^{2}(\Omega)}^{2} \geqslant \int_{\Omega} Q_{\theta}^{\epsilon}(u) \cdot u \mathrm{~d} x \geqslant c \sum_{\alpha, \beta=1}^{K}\left\|u_{\alpha}-u_{\beta}\right\|_{L^{2}(\Omega)}^{2} .
$$


The proof is similar to that of proposition 3.7 in [1]. The collision kernel $Q_{\theta}^{\epsilon}$ is defined by

$$
Q_{\theta}^{\epsilon}(u)=\epsilon J_{\theta}\left(\frac{x}{\epsilon}\right) \cdot \nabla u+\tilde{Q}_{\theta}\left(\frac{x}{\epsilon}\right) u .
$$

Upon defining a second-order tensor $J_{\theta}$ with lines $J_{\theta, \alpha}$,

$$
J_{\theta, \alpha}(y)=A_{\alpha}(y)\left(\psi_{\theta, \alpha}(y) \nabla_{y} \psi_{\theta, \alpha}^{*}(y)-\psi_{\theta, \alpha}^{*}(y) \nabla_{y} \psi_{\theta, \alpha}(y)\right),
$$

and $\tilde{Q}_{\theta}$ is the $Y$-periodic $K \times K$ matrix defined by its entries,

$$
\left.\begin{array}{l}
\tilde{Q}_{\theta, \alpha, \beta}(y)=\left(\Sigma_{\alpha, \beta}(y)-\lambda(\theta) \sigma_{\alpha, \beta}(y)\right) \psi_{\theta, \beta}(y) \psi_{\theta, \alpha}^{*}(y) \leqslant 0 \quad \text { if } \alpha \neq \beta, \\
\tilde{Q}_{\theta, \alpha, \alpha}(y)=-\sum_{\substack{\beta=1 \\
\beta \neq \alpha}}^{K} \tilde{Q}_{\theta, \alpha, \beta}(y)>0 .
\end{array}\right\}
$$

Lemma 3.7. Let $\theta \in U$, with $U$ given by (2.11). Let the linear operator $S_{\theta, \epsilon}$ be defined by

$$
\left.\begin{array}{rl}
S_{\theta, \epsilon}: L^{2}(\Omega)^{K} & \rightarrow L^{2}(\Omega)^{K}, \\
f=\left(f_{\alpha}\right)_{1 \leqslant \alpha \leqslant K} \rightarrow u=\left(u_{\alpha}\right)_{1 \leqslant \alpha \leqslant K} \quad \text { unique solution of } & \\
-\operatorname{div}\left(D_{\theta}\left(\frac{x}{\epsilon}\right) \nabla u\right)+\frac{1}{\epsilon^{2}} Q_{\theta}^{\epsilon}(u)=f \quad \text { in } \Omega, \\
u=0 \quad \text { on } \partial \Omega .
\end{array}\right\}
$$

For any fixed $\epsilon>0, S_{\theta, \epsilon}$ is a linear compact operator in $L^{2}(\Omega)^{K}$.

The homogenization result proved in [1] concerns the compact operator $S_{\theta, \epsilon}$ given by (3.18), for $\theta=0$. To formulate the limit operator $S_{\theta}$ of $S_{\theta, \epsilon}$ as $\epsilon$ goes to zero, we need to introduce the vector-valued corrector functions $\left(\xi_{i}\right)_{1 \leqslant i \leqslant N}$ given by the following lemma.

LEMMA 3.8. Let $\theta \in U$, with $U$ given by (2.11). In $H_{\#}^{1}(Y)^{K}$, define the operator $Q_{\theta}(u)=J_{\theta}(y) \cdot \nabla u+\tilde{Q}_{\theta}(y) u$, where $J_{\theta}$ is given by (3.16) and $\tilde{Q}_{\theta}$ is given by (3.17).

The kernel of $Q_{\theta}$ and of its adjoint $Q_{\theta}^{*}$ are spanned by $\mathbb{1}=\{1, \ldots, 1\} \in \mathbb{R}^{K}$. For all $1 \leqslant i \leqslant N$, if

$$
\int_{Y} Q_{\theta}\left(y_{i}\right) \cdot \mathbb{1}=\sum_{\alpha=1}^{K} \int_{Y} A_{\alpha}(y)\left(\psi_{\theta, \alpha} \nabla \psi_{\theta, \alpha}^{*}-\psi_{\theta, \alpha}^{*} \nabla \psi_{\theta, \alpha}\right) \cdot \nabla y_{i} \mathrm{~d} y=0,
$$

then there is the unique solution $\xi_{i}^{\theta}$ in $H_{\#}^{1}(Y)^{K} /(\mathbb{R} \times \mathbb{1})$ of

$$
\left.\begin{array}{c}
-\operatorname{div}\left(D_{\theta}(y) \nabla\left(\xi_{i}^{\theta}(y)+y_{i} \mathbb{1}\right)\right)+Q_{\theta}\left(\xi_{i}^{\theta}(y)+y_{i} \mathbb{1}\right)=0, \\
y \rightarrow \xi_{i}^{\theta}(y) \quad Y \text {-periodic. }
\end{array}\right\}
$$

Furthermore, condition (3.19) is necessary and sufficient.

REMARK 3.9. This consequence of the Fredholm alternative is exposed in detail in $[1$, lemma 5.2$]$, for $\theta=0$. It can be extended to any $\theta \in U$ without difficulty. For 
$\theta=\theta_{\infty}$, the functions $\left(\xi_{i}^{\infty}\right)_{1 \leqslant i \leqslant N}$ have been defined in two different ways. In theorem 3.3 they are defined as the solutions of system (3.8), whereas in lemma 3.8 they are solutions of system (3.20). Our notation is consistent in the sense that (3.20) is just (3.8), each line being multiplied by $\psi_{\infty, \alpha} \psi_{\infty, \alpha}^{*}$.

Proposition 3.10. Let $f^{\epsilon}$ be a sequence that converges weakly in $L^{2}(\Omega)^{K}$ to $f=\left(f_{\alpha}\right)_{1 \leqslant \alpha \leqslant K}$. Then the sequence $u^{\epsilon}=S_{\theta, \epsilon}\left(f^{\epsilon}\right)$ converges weakly in $H_{0}^{1}(\Omega)^{K}$ to $\left(u^{0}, \ldots, u^{0}\right)$, which is defined by

$$
u^{0}=S_{\theta}\left(\sum_{\alpha=1}^{K} f_{\alpha}\right)
$$

If the symmetry condition (3.19) is not satisfied, then $S_{\theta}=0$. If the symmetry condition (3.19) is satisfied, $S_{\theta}$ is the following compact operator

$$
\begin{aligned}
& S_{\theta}: L^{2}(\Omega) \rightarrow L^{2}(\Omega), \\
& f \rightarrow u \quad \text { unique solution of } \\
& \qquad\left\{\begin{aligned}
-\operatorname{div}\left(\bar{D}_{\theta} \nabla u(x)\right)=f & \text { in } \Omega, \\
u=0 & \text { on } \partial \Omega,
\end{aligned}\right.
\end{aligned}
$$

where $\bar{D}_{\theta}$ is the constant positive definite matrix defined by

$$
\begin{aligned}
\bar{D}_{\theta_{i, j}}=\sum_{\alpha=1}^{K} & \int_{Y} D_{\theta, \alpha}(y) \nabla\left(y_{i}+\xi_{i, \alpha}^{\theta}(y)\right) \cdot \nabla\left(y_{j}+\xi_{j, \alpha}^{\theta}(y)\right) \mathrm{d} y \\
& \quad-\frac{1}{2} \sum_{\alpha, \beta=1}^{K} \int_{Y} \tilde{Q}_{\theta, \alpha, \beta}(y)\left(\xi_{i, \alpha}^{\theta}(y)-\xi_{i, \beta}^{\theta}(y)\right) \cdot\left(\xi_{j, \alpha}^{\theta}(y)-\xi_{j, \beta}^{\theta}(y)\right) \mathrm{d} y .
\end{aligned}
$$

Proof of theorem 3.3. We remark that proposition 3.10 implies that the sequence of operators $S_{\theta, \epsilon}$, defined by (3.18), uniformly converges to the limit operator defined in $L^{2}(\Omega)^{K}$ by

$$
f=\left(f_{\alpha}\right)_{1 \leqslant \alpha \leqslant K} \rightarrow\left(S_{\theta}\left(\sum_{\alpha=1}^{K} f_{\alpha}\right), \ldots, S_{\theta}\left(\sum_{\alpha=1}^{K} f_{\alpha}\right)\right) .
$$

The asymptotic analysis of the eigenvalue problem (3.10) is truly controlled by the convergence of the sequence of operators $T_{\theta, \epsilon}$ defined by

$$
\begin{gathered}
T_{\theta, \epsilon}: L^{2}(\Omega)^{K} \rightarrow L^{2}(\Omega)^{K}, \\
f=\left(f_{\alpha}\right)_{1 \leqslant \alpha \leqslant K} \rightarrow S_{\theta, \epsilon}\left(B\left(\frac{x}{\epsilon}\right) f\right) .
\end{gathered}
$$

Namely, the eigenvalues of $T_{\theta, \epsilon}$ are inverse of those of (3.10). Introducing the averages

$$
\bar{B}_{\alpha, \beta}=\int_{Y} B_{\alpha, \beta}(y) \mathrm{d} y
$$


which are the weak limits of the entries of the matrix $B(x / \epsilon)$, we define a limit operator $T_{\theta}$ by

$$
\begin{aligned}
T_{\theta}: L^{2}(\Omega)^{K} & \rightarrow L^{2}(\Omega)^{K} \\
f=\left(f_{\alpha}\right)_{1 \leqslant \alpha \leqslant K} & \rightarrow\left(S_{\theta}\left(\sum_{\alpha, \beta=1}^{K} \bar{B}_{\alpha, \beta} f_{\beta}\right), \ldots, S_{\theta}\left(\sum_{\alpha, \beta=1}^{K} \bar{B}_{\alpha, \beta} f_{\beta}\right)\right) .
\end{aligned}
$$

The sequence $T_{\theta, \epsilon}$ converges pointwise to $T_{\theta}$, but usually not uniformly. However, proposition 3.10 implies that the sequence of operators $T_{\theta, \epsilon}$ is collectively compact (see, for example, $[4,8]$ ). A consequence is that the $m$ th eigenvalue of $T_{\theta, \epsilon}$ converges to the $m$ th eigenvalue of $T_{\theta}$ (counted with their multiplicity). If $S_{\theta}=0$, $T_{\theta, \epsilon}$ converges to 0 , and so does all its eigenvalues. If $S_{\theta} \not \equiv 0$, which means, by proposition 3.10 ,

$$
\sum_{\alpha=1}^{K} \int_{Y} A_{\alpha}(y)\left(\psi_{\theta, \alpha} \nabla \psi_{\theta, \alpha}^{*}-\psi_{\theta, \alpha}^{*} \nabla \psi_{\theta, \alpha}\right) \cdot \nabla y_{i} \mathrm{~d} y=0 \quad \text { for all } 1 \leqslant i \leqslant N,
$$

i.e. $\theta=\theta_{\infty}$, because of the characterization (2.12), we obtain theorem 3.3.

\section{Existence of a maximum drift eigenvalue}

This section is devoted to the proof of theorem 2.8. We shall proceed in three steps. First, we shall prove the result in the particular case when the right-hand side $\sigma=I_{K}$. A consequence will be that the domain $U$ defined by (2.11) will be shown to be bounded. We will then turn to the characteristic property (2.12) of the maximum eigen-triplet $\left(\lambda_{\infty}, \psi_{\infty}, \psi_{\infty}^{*}\right)$. Finally, we will prove the uniqueness of such a triplet.

The notation we shall use in this section will be the following. The bracket product $\langle\cdot\rangle$ is the scalar product in $L^{2}(Y)^{K}$, i.e.

$$
\langle u, v\rangle=\sum_{\alpha=1}^{K} \int_{Y} u_{\alpha}(y) v_{\alpha}(y) \mathrm{d} y \quad \forall u, v \in L^{2}(Y)^{K} .
$$

We shall also use it for second-order operators such as $A(\theta)$, and $\left(\partial A / \partial \theta_{i}\right)(\theta)$, i.e. for all $\phi, \psi \in H_{\#}^{1}(Y)^{K}$,

$$
\begin{aligned}
\langle A(\theta) \phi, \psi\rangle & =\sum_{\alpha=1}^{K} \sum_{i, j=1}^{N} \int_{Y} A_{\alpha, i, j}(y)\left(\frac{\partial \phi_{\alpha}}{\partial y_{j}}+\theta_{j} \phi_{\alpha}\right) \cdot\left(\frac{\partial \psi_{\alpha}}{\partial y_{i}}-\theta_{i} \psi_{\alpha}\right) \mathrm{d} y, \quad \text { (4.1) } \\
\left\langle\frac{\partial A}{\partial \theta_{i}}(\theta) \phi, \psi\right\rangle=\sum_{\alpha=1}^{K} \sum_{j=1}^{N} \int_{Y} A_{\alpha, i, j}(y) & \\
& \times\left(\phi_{\alpha} \cdot\left(\frac{\partial \psi_{\alpha}}{\partial y_{j}}-\theta_{j} \psi_{\alpha}\right)-\left(\frac{\partial \phi_{\alpha}}{\partial y_{j}}+\theta_{j} \phi_{\alpha}\right) \cdot \psi_{\alpha}\right) \mathrm{d} y .
\end{aligned}
$$


STEP 1 (Special case $\sigma=I_{K}$ ). Let us first consider system (2.10), similar to (1.4), but with identity as a right-hand side,

$$
\left.\begin{array}{c}
A(\theta) \varphi_{\theta}+\Sigma \varphi_{\theta}=\Lambda(\theta) \varphi_{\theta}, \\
y \rightarrow \varphi_{\theta}(y) \quad Y \text {-periodic, } \\
\vartheta_{\theta}=\mathrm{e}^{\theta \cdot y} \varphi(y) .
\end{array}\right\}
$$

The adjoint equation of (4.3) defining $\vartheta_{\theta}^{*}$ is given by

$$
\left.\begin{array}{c}
A^{*}(\theta) \varphi_{\theta}+\Sigma^{*} \varphi_{\theta}=\Lambda(\theta) \varphi_{\theta}, \\
y \rightarrow \varphi_{\theta}(y) \quad Y \text {-periodic, } \\
\vartheta_{\theta}=\mathrm{e}^{-\theta \cdot y} \varphi(y) .
\end{array}\right\}
$$

As indicated in remark 2.3, the eigenvectors are normalized as follows:

$$
\left\langle\varphi_{\theta}, \varphi_{\theta}\right\rangle=1 \text { and }\left\langle\varphi_{\theta}, \varphi_{\theta}^{*}\right\rangle=1
$$

In this simple case, theorem 2.8 is a straightforward consequence of the following lemma.

LEMMA 4.1. Let $\Lambda(\theta)$ be the first eigenvalue of system (4.3). We have the following relation:

$$
\frac{\partial \Lambda}{\partial \theta_{i}}(\theta)=\sum_{\alpha=1}^{K} \int_{Y} A_{\alpha}(y)\left(\vartheta_{\theta, \alpha}(y) \nabla \vartheta_{\theta, \alpha}^{*}(y)-\vartheta_{\theta, \alpha}^{*}(y) \nabla \vartheta_{\theta, \alpha}(y)\right) \cdot \nabla y_{i} \mathrm{~d} y
$$

Furthermore, the application $\theta \rightarrow \Lambda(\theta) \in C^{\infty}\left(\mathbb{R}^{N}\right)$ is strictly concave, i.e. for all $\theta \in \mathbb{R}^{N}$, there exists a constant $C(\theta)>0$

$$
\forall \zeta \in \mathbb{R}^{N}, \quad-\sum_{i, j=1}^{N} \frac{\partial^{2} \Lambda}{\partial \theta_{i} \partial \theta_{j}} \zeta_{j} \cdot \zeta_{i} \geqslant C(\theta) \sum_{i=1}^{N} \zeta_{i} \cdot \zeta_{i}
$$

and for all $U \subset \mathbb{R}^{N}$, if $\min _{U} \Lambda(\theta) \neq-\infty$, then $\min _{U} C(\theta)>0$.

As a consequence, $\lim _{|\theta| \rightarrow \infty} \Lambda(\theta)=-\infty$ and the domain $U=\Lambda^{-1}\left(\mathbb{R}_{+}^{*}\right)$ is a bounded open connected subset of $\mathbb{R}^{N}$.

Proof. Let us formally differentiate the above equation defining $\varphi_{\theta}$ with respect to $\theta_{i}$, the $i$ th component of vector $\theta$,

$$
\frac{\partial A}{\partial \theta_{i}}(\theta) \varphi_{\theta}+A(\theta) \frac{\partial \varphi_{\theta}}{\partial \theta_{i}}+\Sigma \frac{\partial \varphi_{\theta}}{\partial \theta_{i}}=\frac{\partial \Lambda}{\partial \theta_{i}}(\theta) \varphi_{\theta}+\Lambda(\theta) \frac{\partial \varphi_{\theta}}{\partial \theta_{i}} .
$$

The above expression, tested variationally against $\varphi_{\theta}^{*}$, given by (4.4), gives

$$
\frac{\partial \Lambda}{\partial \theta_{i}}(\theta)=\frac{\partial \Lambda}{\partial \theta_{i}}(\theta)\left\langle\varphi_{\theta}, \varphi_{\theta}^{*}\right\rangle=\left\langle\frac{\partial A}{\partial \theta_{i}}(\theta) \varphi_{\theta}, \varphi_{\theta}^{*}\right\rangle
$$


which writes explicitly as follows:

$$
\begin{aligned}
\frac{\partial \Lambda}{\partial \theta_{i}}(\theta) & =\sum_{\alpha=1}^{K} \sum_{j=1}^{N} \int_{Y} A_{\alpha, i, j}(y)\left(-\varphi_{\theta}^{*} \frac{\partial \varphi_{\theta}}{\partial y_{j}}+\varphi_{\theta} \frac{\partial \varphi_{\theta}^{*}}{\partial y_{j}}-2 \varphi_{\theta} \varphi_{\theta}^{*} \theta_{j}\right) \mathrm{d} y \\
& =\sum_{\alpha=1}^{K} \int_{Y} A_{\alpha}(y)\left(\vartheta_{\theta, \alpha}(y) \nabla \vartheta_{\theta, \alpha}^{*}(y)-\vartheta_{\theta, \alpha}^{*}(y) \nabla \vartheta_{\theta, \alpha}(y)\right) \cdot \nabla y_{i} \mathrm{~d} y .
\end{aligned}
$$

The right-hand side is well defined, and gives formula (4.6) for $\partial \Lambda / \partial \theta_{i}$. Because of the algebraic and geometric simplicity of $\Lambda(\theta)$, equation (4.8) is, in fact, a necessary and sufficient condition of existence and uniqueness of $\partial \varphi_{\theta} / \partial \theta_{i}$ given by (4.7).

More precisely, if we introduce the change of variable

$$
\Xi_{i, \alpha}^{\theta}=\left(\frac{\partial \varphi_{\theta}}{\partial \theta_{i}}\right)_{\alpha} \frac{1}{\varphi_{\theta, \alpha}},
$$

we see that $\Xi_{i}^{\theta}$ is the unique solution of the following system,

$$
\left.\begin{array}{c}
-\operatorname{div}\left(D_{\theta}(y) \nabla\left(\Xi_{i}^{\theta}(y)+y_{i} \mathbb{1}\right)\right)+Q_{\theta}\left(\Xi_{i}^{\theta}(y)+y_{i} \mathbb{1}\right)=\frac{\partial \Lambda}{\partial \theta_{i}}(\theta) \vartheta_{\theta} \cdot\left(\vartheta_{\theta}^{*}\right)^{\mathrm{T}}, \\
y \rightarrow \Xi_{i}^{\theta}(y) \quad Y \text {-periodic, }
\end{array}\right\}
$$

where $D_{\theta}$ is given by

$$
D_{\theta, \alpha}(y)=\vartheta_{\theta, \alpha}(y) \vartheta_{\theta, \alpha}^{*}(y) A_{\alpha}(y) \quad \forall \alpha \in 1, \ldots, K,
$$

which corresponds to formula (3.12), and $Q_{\theta}$ is given by

$$
Q_{\theta}(u)=J_{\theta}(y) \cdot \nabla u+\tilde{Q}_{\theta}(y) u .
$$

Upon defining a second-order tensor $J_{\theta}$ with lines $J_{\theta, \alpha}$,

$$
J_{\theta, \alpha}(y)=A_{\alpha}(y)\left(\vartheta_{\theta, \alpha}(y) \nabla_{y} \vartheta_{\theta, \alpha}^{*}(y)-\vartheta_{\theta, \alpha}^{*}(y) \nabla_{y} \vartheta_{\theta, \alpha}(y)\right),
$$

and $\tilde{Q}_{\theta}$, the $Y$-periodic $K \times K$ matrix defined by its entries

$$
\left.\begin{array}{l}
\tilde{Q}_{\theta, \alpha, \beta}(y)=\left(\Sigma_{\alpha, \beta}(y)-\Lambda(\theta)\right) \vartheta_{\theta, \beta}(y) \vartheta_{\theta, \alpha}^{*}(y) \text { if } \alpha \neq \beta, \\
\tilde{Q}_{\theta, \alpha, \alpha}(y)=-\sum_{\substack{\beta=1 \\
\beta \neq \alpha}}^{K} \tilde{Q}_{\theta, \alpha, \beta}(y) .
\end{array}\right\}
$$

Formula (4.12) corresponds to (3.15), with $\sigma=I_{K}$. Thus lemma 3.8 gives the existence and uniqueness of $\Xi_{i}^{\theta} \in H_{\#}^{1}(Y)^{K} /(\mathbb{R} \times \mathbb{1})$, provided that $(4.9)$ is satisfied. Consequently, the formal differentiation performed in (4.7) was licit.

In a similar manner, we obtain that $\partial \varphi_{\theta}^{*} / \partial \theta_{j}$ exists and is given by

$$
\left(\frac{\partial \varphi_{\theta}^{*}}{\partial \theta_{i}}\right)_{\alpha}=\varphi_{\theta, \alpha}^{*} \Xi_{i, \alpha}^{\theta, *}
$$


where $\Xi_{i}^{\theta, *}$ is a solution of the following system:

$$
\left.\begin{array}{c}
-\operatorname{div}\left(D_{\theta}(y) \nabla\left(\Xi_{i}^{\theta, *}(y)-y_{i} \mathbb{1}\right)\right)+Q_{\theta}^{*}\left(\Xi_{i}^{\theta, *}(y)-y_{i} \mathbb{1}\right)=\frac{\partial \Lambda}{\partial \theta_{i}}(\theta) \phi_{\theta}\left(\phi_{\theta}^{*}\right)^{\mathrm{T}}, \\
y \rightarrow \Xi_{i}^{\theta, *}(y) \quad Y \text {-periodic. }
\end{array}\right\}
$$

Let us now differentiate (4.8) with respect to $\theta_{j}$, the $j$ th component of vector $\theta$,

$$
\frac{\partial^{2} \Lambda}{\partial \theta_{i} \partial \theta_{j}}(\theta)=\left\langle\frac{\partial A}{\partial \theta_{i} \partial \theta_{j}}(\theta) \varphi_{\theta}, \varphi_{\theta}^{*}\right\rangle+\left\langle\frac{\partial A}{\partial \theta_{i}}(\theta) \frac{\partial \varphi_{\theta}}{\partial \theta_{j}}, \varphi_{\theta}^{*}\right\rangle+\left\langle\frac{\partial A}{\partial \theta_{i}}(\theta) \varphi_{\theta}, \frac{\partial \varphi_{\theta}^{*}}{\partial \theta_{j}}\right\rangle .
$$

Since every term of the right-hand side of (4.16) is well defined, we have obtained a formula for $\partial^{2} \Lambda / \partial \theta_{i} \partial \theta_{j}$. After some algebraic manipulations similar to that done in $[1$, propositions $5.6,5.7]$, we obtain that it can be written also under the following equivalent forms,

$$
\frac{\partial^{2} \Lambda}{\partial \theta_{i} \partial \theta_{j}}=-\left(\bar{D}_{\theta, i, j}+\bar{D}_{\theta, i, j}^{*}\right)=-2 \bar{D}_{\theta, i, j}=-2 \bar{D}_{\theta, i, j}^{*}
$$

where the matrix $\bar{D}_{\theta}$ is defined by its entries with the help of the corrector functions $\left(\Xi_{i}^{\theta}\right)_{1 \leqslant i \leqslant N}$,

$$
\begin{aligned}
\bar{D}_{\theta, i, j}=\sum_{\alpha=1}^{K} \int_{Y} D_{\theta, \alpha}(y) \nabla\left(y_{i}+\Xi_{i, \alpha}^{\theta}(y)\right) \cdot \nabla\left(y_{j}+\Xi_{j, \alpha}^{\theta}(y)\right) \mathrm{d} y \\
\quad-\frac{1}{2} \sum_{\alpha, \beta=1}^{K} \int_{Y} \tilde{Q}_{\theta, \alpha, \beta}(y)\left(\Xi_{i, \alpha}^{\theta}(y)-\Xi_{i, \beta}^{\theta}(y)\right) \cdot\left(\Xi_{j, \alpha}^{\theta}(y)-\Xi_{j, \beta}^{\theta}(y)\right) \mathrm{d} y
\end{aligned}
$$

and the matrix $\bar{D}_{\theta}^{*}$ is defined similarly, with the help of the corrector functions $\left(\Xi_{i}^{\theta, *}\right)_{1 \leqslant i \leqslant N}$

$$
\begin{aligned}
\bar{D}_{\theta, i, j}^{*}=\sum_{\alpha=1}^{K} \int_{Y} D_{\theta, \alpha}(y) \nabla\left(y_{i}-\Xi_{i, \alpha}^{\theta, *}(y)\right) \cdot \nabla\left(y_{j}-\Xi_{j, \alpha}^{\theta, *}(y)\right) \mathrm{d} y \\
\quad-\frac{1}{2} \sum_{\alpha, \beta=1}^{K} \int_{Y} \tilde{Q}_{\theta, \alpha, \beta}(y)\left(\Xi_{i, \alpha}^{\theta, *}(y)-\Xi_{i, \beta}^{\theta, *}(y)\right) \cdot\left(\Xi_{j, \alpha}^{\theta, *}(y)-\Xi_{j, \beta}^{\theta, *}(y)\right) \mathrm{d} y .
\end{aligned}
$$

To conclude the proof, let us now show that $\bar{D}_{\theta}$ is positive definite,

$$
\begin{array}{r}
\sum_{i, j=1}^{N} \bar{D}_{\theta, i, j} \xi_{i} \xi_{j}=\sum_{\alpha=1}^{K} \sum_{i, j=1}^{N} \int_{Y} D_{\theta, \alpha}(y) \nabla\left(y_{i}+\Xi_{i, \alpha}^{\theta}(y)\right) \cdot \nabla\left(y_{j}+\Xi_{j, \alpha}^{\theta}(y)\right) \zeta_{i} \zeta_{j} \mathrm{~d} y \\
-\frac{1}{2} \sum_{\alpha, \beta=1}^{K} \sum_{i, j=1}^{N} \int_{Y} \tilde{Q}_{\theta, \alpha, \beta}(y)\left(\Xi_{i, \alpha}^{\theta}(y)-\Xi_{i, \beta}^{\theta}(y)\right) \\
\cdot\left(\Xi_{j, \alpha}^{\theta}(y)-\Xi_{j, \beta}^{\theta}(y)\right) \zeta_{i} \zeta_{j} \mathrm{~d} y
\end{array}
$$




$$
\begin{aligned}
\geqslant & \sum_{\alpha=1}^{K} \sum_{i, j=1}^{N} \int_{Y} D_{\theta, \alpha}(y) \nabla\left(y_{i}+\Xi_{i, \alpha}^{\theta}(y)\right) \cdot \nabla\left(y_{j}+\Xi_{j, \alpha}^{\theta}(y)\right) \zeta_{i} \zeta_{j} \mathrm{~d} y \\
& -\frac{1}{2} \sum_{\alpha, \beta=1}^{K} \int_{Y} \tilde{Q}_{\alpha, \beta}(y)\left(\sum_{i=1}^{N}\left(\Xi_{i, \alpha}^{\theta}-\Xi_{i, \beta}^{\theta}\right) \xi_{i}\right)^{2} \mathrm{~d} y .
\end{aligned}
$$

Since $\tilde{Q}_{\alpha, \beta} \leqslant 0$ for all $\alpha \neq \beta$, the second term if positive. Thus

$$
\begin{aligned}
\sum_{i, j=1}^{N} \bar{D}_{\theta, i, j} \xi_{i} \xi_{j} & \geqslant \sum_{\alpha=1}^{K} \min _{w \in H_{\#}^{1}(y)} \int_{Y} \varphi_{\theta, \alpha} \varphi_{\theta, \alpha}^{*} A_{\alpha}(y)(\nabla w(y)+\zeta) \cdot(\nabla w(y)+\zeta) \mathrm{d} y \\
& \geqslant C|\xi|^{2} \sum_{\alpha=1}^{K}\left(\int_{Y} \frac{1}{\varphi_{\theta, \alpha}(y) \varphi_{\theta, \alpha}^{*}(y)} \mathrm{d} y\right)^{-1}
\end{aligned}
$$

where $C>0$ is the coercivity constant of $A$ introduced in (2.2). This concludes the proof of the strict concavity of $\Lambda$ for all $\theta$ in $\mathbb{R}^{N}$, if we define

$$
C(\theta)=C\left(\int_{Y} \frac{1}{\varphi_{\theta, \alpha}(y) \varphi_{\theta, \alpha}^{*}(y)} \mathrm{d} y\right)^{-1} .
$$

Let us now show that for any $U \subset \mathbb{R}^{N}$, if $\min _{U} \lambda(\theta) \neq-\infty$, then $\min _{U} C(\theta)>0$. Suppose we can choose $\infty>c>0$ such that $-c<\min _{U} \Lambda(\theta)$. By definition, for any $\theta \in U$, we have

$$
-\operatorname{div}\left(A_{\alpha}(y) \nabla \vartheta_{\theta, \alpha}\right)+\Sigma_{\alpha \alpha}(y) \vartheta_{\theta, \alpha}=\Lambda(\theta) \vartheta_{\theta, \alpha}-\sum_{\beta=2}^{K} \Sigma_{\alpha \beta} \vartheta_{\theta, \beta},
$$

and since $\Sigma_{\alpha, \beta} \leqslant 0$ for $\alpha \neq \beta$, this implies that, for any $\alpha$ and $\theta \in U$,

$$
-\operatorname{div}\left(A_{\alpha}(y) \nabla \vartheta_{\theta, \alpha}\right)+\left(c+\Sigma_{\alpha, \alpha}(y)\right) \vartheta_{\theta, \alpha}>0 .
$$

Since $\vartheta_{\theta, \alpha} \in W_{\text {loc }}^{1,2}\left(\mathbb{R}^{N}\right)$ and $\vartheta_{\theta, \alpha}>0$, this implies, by a weak Harnack inequality (see $[10$, p. 184]), that

$$
\min _{Y} \vartheta_{\theta, \alpha} \geqslant m\left\|\vartheta_{\theta, \alpha}\right\|_{L^{2}(2 Y)}
$$

where $m>0$ depends on $c, A$ and $\Sigma$, but is independent of $\theta$ and $\alpha$. The same argument applies for $\vartheta_{\theta, \alpha}^{*}$, for all $\alpha$. Consequently,

$$
\begin{aligned}
\sum_{\alpha=1}^{K} \inf _{Y}\left(\vartheta_{\theta, \alpha} \vartheta_{\theta, \alpha}^{*}\right) & >m^{2} \sum_{\alpha=1}^{K}\left\|\vartheta_{\theta, \alpha}\right\|_{L^{2}(2 Y)}\left\|\vartheta_{\theta, \alpha}^{*}\right\|_{L^{2}(2 Y)} \\
& >m^{2} \sum_{\alpha=1}^{K} \int_{2 Y} \vartheta_{\theta, \alpha} \vartheta_{\theta, \alpha}^{*} \\
& =2 m^{2}\left\langle\varphi_{\theta}, \varphi_{\theta}^{*}\right\rangle \\
& =2 m^{2}
\end{aligned}
$$

and therefore $\min _{U} C(\theta)>2 K C m^{2}>0$. 
STEP 2 (General case). The results of lemma 4.1 can be partly extended to the general case when $\sigma \not \equiv I_{K}$, as is shown by the following lemma.

LEMmA 4.2. Let $\theta \in U$ be fixed, and let $\lambda(\theta)$ be the first eigenvalue of system (1.4). We have the following relation:

$$
\frac{\partial \lambda}{\partial \theta_{i}}(\theta)=\sum_{\alpha=1}^{K} \int_{Y} A_{\alpha}(y)\left(\psi_{\theta, \alpha}(y) \nabla \psi_{\theta, \alpha}^{*}(y)-\psi_{\theta, \alpha}^{*}(y) \nabla \psi_{\theta, \alpha}(y)\right) \cdot \nabla y_{i} \mathrm{~d} y
$$

Proof. The proof of this result is very similar to that of lemma 4.1. Formula (4.21) is an explicit formulation of the identity (4.8), with the normalization

$$
\left\langle\sigma \psi_{\theta}, \psi_{\theta}^{*}\right\rangle=1
$$

which was introduced in remark 2.3.

Lemma 4.3. Let $\theta_{0} \in U$ be fixed. There exist $U^{\prime}$, an open connected subset of $U$ such that $\bar{u}^{\prime} \subset U$, and $\lambda(\theta)<\lambda\left(\theta_{0}\right)$ in $U \backslash \bar{u}^{\prime}$.

Proof. For any $\theta \in U$, if we factorize the positive normalized eigensolution $\psi_{\theta}$ of the eigenvalue problem (1.4) by the cell eigenvector $\psi_{\theta_{0}}$ of the eigenvalue problem (1.3) for $\theta=\theta_{0}$ in the following way,

$$
\psi_{\theta, \alpha}=\psi_{\theta_{0}, \alpha} \phi_{\theta, \alpha}
$$

then $\phi_{\theta}$ is a positive eigenvector of the following eigenvalue problem,

$$
\left.\begin{array}{c}
-\operatorname{div}\left(D_{0} \nabla \phi_{\theta}\right)+Q_{0}\left(\phi_{\theta}\right)=\mu(\theta) B_{0} \phi_{\theta} \quad \text { in } Y, \\
\phi_{\theta} \exp \left(-\left(\theta-\theta_{0}\right) \cdot y\right) \quad Y \text {-periodic, }
\end{array}\right\}
$$

where $\mu(\theta)=\lambda(\theta)-\lambda\left(\theta_{0}\right), D_{0}=D_{\theta_{0}}$ given by (3.12), $Q_{0}=Q_{\theta_{0}}$ given by (3.15) and $B_{0}=B_{\theta_{0}}$ given by (3.13). Under this form, this first positive direct and adjoint eigenvectors $\phi_{\theta}$ and $\phi_{\theta}^{*}$ are constant and equal to $\mathbb{1}=(1, \ldots, 1)$ for $\theta=\theta_{0}$, and $\mu\left(\theta_{0}\right)=0$. Replacing $B_{0}$ by $I_{K}$, we obtain the following eigenvalue problem:

$$
\left.\begin{array}{cc}
-\operatorname{div}\left(D_{0} \nabla \varphi_{\theta}\right)+Q_{0}\left(\varphi_{\theta}\right)=M(\theta) \varphi_{\theta} & \text { in } Y, \\
\varphi_{\theta} \exp (-\theta \cdot y) \quad Y \text {-periodic. } &
\end{array}\right\}
$$

As before, $M(\theta)$ is a $C^{\infty}$ function from $\mathbb{R}^{N}$ into $\mathbb{R}$. Proposition 2.7 shows that if $M(\theta)>0$, then $\mu(\theta)>0$. Conversely, if $\mu(\theta)>0$, then $-\operatorname{div}\left(D_{0} \nabla \phi_{\theta}\right)+Q_{0}\left(\phi_{\theta}\right) \geqslant 0$ and thus $\phi$ is a positive supersolution of (4.24). Theorem 2.6 then applies and $M(\theta)>0$. We have obtained that $\mu^{-1}\left(\mathbb{R}_{+}^{*}\right)$ is also $U^{\prime}=M^{-1}\left(\mathbb{R}_{+}^{*}\right)$. By a similar argument, $U=\lambda^{-1}\left(\mathbb{R}_{+}^{*}\right)$, thus $U^{\prime} \subset U$, since $\mu(\theta)=\lambda(\theta)-\lambda\left(\theta_{0}\right)$ and $\lambda\left(\theta_{0}\right)>0$.

Consequently, if we can prove that $U^{\prime}$ is a connected open subset of $\mathbb{R}^{N}$, then, for all $\theta \in U \backslash \bar{u}^{\prime}, \mu(\theta)<0$ and the lemma is proved.

In a similar manner as in the proof of lemma 4.1, we obtain that

$$
\frac{\partial^{2} M}{\partial \theta_{i} \partial \theta_{j}}(\theta)=-2 \bar{D}_{\theta, i, j}^{\prime}
$$


where $\bar{D}_{\theta, i, j}^{\prime}$ is defined by its entries with the help of the functions $\left(T_{i}^{\theta}\right)_{1 \leqslant i \leqslant N}$,

$$
\begin{aligned}
\bar{D}_{\theta, i, j}^{\prime}=\sum_{\alpha=1}^{K} \int_{Y} D_{\theta, \alpha}^{\prime}(y) \nabla\left(y_{i}+T_{i, \alpha}^{\theta}(y)\right) \cdot \nabla\left(y_{j}+T_{j, \alpha}^{\theta}(y)\right) \mathrm{d} y \\
\quad-\frac{1}{2} \sum_{\alpha, \beta=1}^{K} \int_{Y} \tilde{Q}_{\theta, \alpha, \beta}^{\prime}(y)\left(T_{i, \alpha}^{\theta}(y)-T_{i, \beta}^{\theta}(y)\right) \cdot\left(T_{j, \alpha}^{\theta}(y)-T_{j, \beta}^{\theta}(y)\right) \mathrm{d} y .
\end{aligned}
$$

Here, the new diffusion matrices $D_{\theta}^{\prime}$ are given by

$$
D_{\theta, \alpha, i, j}^{\prime}(y)=\phi_{\theta, \alpha}(y) \phi_{\theta, \alpha}^{*}(y) D_{0, \alpha, i, j}(y), \quad 1 \leqslant \alpha \leqslant K, \quad 1 \leqslant i, \quad j \leqslant N,
$$

the $Y$-periodic $K \times K$ matrix $\tilde{Q}_{\theta}^{\prime}$ is defined by its entries

$$
\left.\begin{array}{l}
\tilde{Q}_{\theta, \alpha, \beta}^{\prime}(y)=\tilde{Q}_{0, \alpha, \beta}(y) \phi_{\theta, \beta}(y) \phi_{\theta, \alpha}^{*}(y) \quad \text { if } \alpha \neq \beta, \\
\tilde{Q}_{\theta, \alpha, \alpha}^{\prime}(y)=-\sum_{\substack{\beta=1 \\
\beta \neq \alpha}}^{K} \tilde{Q}_{\theta, \alpha, \beta}^{\prime}(y),
\end{array}\right\}
$$

and the auxiliary function $T_{i}^{\theta}$ is a solution of the following system,

$$
\left.\begin{array}{rl}
-\operatorname{div}\left(D_{\theta}^{\prime}(y) \nabla\left(T_{i}^{\theta}(y)-y_{i} \mathbb{1}\right)\right)+J_{\theta}^{\prime} \cdot \nabla\left(T_{i}^{\theta}(y)-y_{i} \mathbb{1}\right)+\tilde{Q}_{\theta}^{\prime} T_{i}^{\theta}(y) \\
=\frac{\sum_{\alpha=1}^{K} \int_{Y} J_{\theta, \alpha}(y) \cdot e_{i} \mathrm{~d} y}{\sum_{\alpha=1}^{K} \int_{Y} \phi_{\theta, \alpha}(y) \phi_{\theta, \alpha}^{*}(y) \mathrm{d} y} \phi_{\theta}\left(\phi_{\theta}^{*}\right)^{\mathrm{T}}, \\
y \rightarrow T_{i}^{\theta}(y) \quad Y \text {-periodic, }
\end{array}\right\}
$$

where the second-order tensor $J_{\theta}^{\prime}$, with lines $J_{\theta, \alpha}^{\prime}$, is defined by

$$
J_{\theta, \alpha}^{\prime}(y)=D_{0, \alpha}(y)\left(\phi_{\theta, \alpha}(y) \nabla_{y} \phi_{\theta, \alpha}^{*}(y)-\phi_{\theta, \alpha}^{*}(y) \nabla_{y} \phi_{\theta, \alpha}(y)\right)+J_{0, \alpha} \phi_{\theta, \alpha} \phi_{\theta, \alpha}^{*} .
$$

Since the eigensolutions $\left(\psi_{\theta_{0}}, \psi_{\theta_{0}}^{*}\right)$ of the periodic cell problems (1.4) and (2.8) are continuous and strictly positive, the uniform coercivity of $A$ in (2.2) induces that of $D_{0}$. Thus, as in the proof of lemma 4.1, we obtain from (4.25) that

$$
\forall \zeta \in \mathbb{R}^{N}, \quad-\sum_{i, j=1}^{N} \frac{\partial^{2} M}{\partial \theta_{i} \partial \theta_{j}}(\theta) \zeta_{i} \zeta_{j} \geqslant C \sum_{i=1}^{N}\left|\zeta_{i}\right|^{2}
$$

and $\lim _{|\theta| \rightarrow \infty} M(\theta)=-\infty$. As a consequence, $U^{\prime}=M^{-1}\left(\mathbb{R}_{+}^{*}\right)$ is a bounded connected open subset of $\mathbb{R}^{N}$.

Proof of theorem 2.8. By lemma 4.3, the continuous application $\lambda \rightarrow \lambda(\theta)$ does not admit a minimum in $U$. Furthermore, for any $\theta_{0} \in U$, the set

$$
\left\{\theta \in U \text { such that } \lambda(\theta) \geqslant \lambda\left(\theta_{0}\right)\right\}
$$

is connected, thus there exists only one extremum value of $\lambda(\theta)$, which is reached on a closed connected subset of $U$. It is simple to prove by a contradiction argument 
that such a subset must be reduced to a point, $\theta_{\infty}$. Using identity (4.21), it satisfies

$$
\sum_{\alpha=1}^{K} \int_{Y} A_{\alpha}(y)\left(\psi_{\theta_{\infty}, \alpha}(y) \nabla \psi_{\theta_{\infty}, \alpha}^{*}(y)-\psi_{\theta_{\infty}, \alpha}^{*}(y) \nabla \psi_{\theta_{\infty}, \alpha}(y)\right)=0 .
$$

REMARK 4.4. In lemma 4.2, at the difference of lemma 4.1, no mention is made of the fact that $\theta \rightarrow \lambda(\theta)$ is concave. This may not be true in the general case (see formula (5.3)), although we have not been able to provide a counter example. We therefore had to use the concavity of the auxiliary eigenvalue $M(\theta)$ to show the one bump shape of the graph of $\lambda(\theta)$ on $U$.

\section{Approximate computational formulae}

The limiting aspect of the above results is that the drift factor $\theta_{\infty}$ is not given by an explicit equation, but may only be obtained through the maximization of the one bump function $\theta \rightarrow \lambda(\theta)$, which usually implies an iterative procedure. Besides, the boundary conditions of the eigenvalue problem (1.4) defining $\lambda(\theta)$ are $\theta$ dependent, and although it can be reformulated as an eigenvalue problem on a fixed domain with $\theta$ dependent coefficients, this tends to complicate the computation. Therefore, when numerical values for the homogenized coefficients are computed, it is natural to evaluate first if the drift-free formulae (3.2) and (3.3) can be used. Then, whether the drift phenomenon must be taken into account or not is revealed by the calculation of the compatibility condition (3.1), that is, when

$$
J=\sum_{\alpha=1}^{K} \int_{Y} A_{\alpha}(y)\left(\psi_{\alpha} \nabla \psi_{\alpha}^{*}-\psi_{\alpha}^{*} \nabla \psi_{\alpha}\right) \mathrm{d} y \neq 0
$$

where $\left(\psi_{\alpha}\right)_{1 \leqslant \alpha \leqslant K}$ (respectively, $\left(\psi_{\alpha}^{*}\right)_{1 \leqslant \alpha \leqslant K}$ ) are the components of the first eigenvector $\psi$ of $(1.3)$ (respectively, $\psi^{*}$ of $(2.7)$ ). In this section, we show that the drift phenomenon is controlled by the modulus of $J$ given by (5.1). More precisely, for small values of $J$, we give asymptotic formulae for $\theta_{\infty}, \psi_{\infty}$ and $D_{\infty}$, which can be obtained with the same amount of computation as in the drift-free case $\left(\theta_{\infty}=0\right)$.

Recall that the eigenfunctions $\left(\psi, \psi^{*}\right)$ of the periodic cell problem are given by systems (1.3) and (2.7). Recall also that the eigenvectors are normalized in the following way:

$$
\sum_{\alpha=1}^{K} \int_{Y} \psi_{\alpha}(y) \cdot \psi_{\alpha}(y) \mathrm{d} y=1 \quad \text { and } \quad \sum_{\alpha, \beta=1}^{K} \int_{Y} \sigma_{\alpha, \beta}(y) \psi_{\beta}(y) \cdot \psi_{\alpha}^{*}(y) \mathrm{d} y=1 .
$$

Then it is proved in lemma 4.2 that the gradient of $\lambda(\theta)$ at $\theta=0$ is given by

$$
\frac{\partial \lambda}{\partial \theta_{i}}(0)=J_{i}=\sum_{\alpha=1}^{K} \int_{Y} A_{\alpha}(y)\left(\psi_{\alpha}(y) \nabla \psi_{\alpha}^{*}(y)-\psi_{\alpha}^{*}(y) \nabla \psi_{\alpha}(y)\right) \cdot \nabla y_{i} \mathrm{~d} y .
$$


After some algebra, a formula comparable to that obtained for the Hessian matrix of $\Lambda(\theta)$ in (4.17) can be derived. It writes as follows,

$$
\begin{aligned}
-\frac{1}{2} \frac{\partial^{2} \lambda}{\partial \theta_{i} \partial \theta_{j}}(0)= & \bar{D}_{i, j} \\
= & \sum_{\alpha=1}^{K} \int_{Y} \psi_{\alpha}(y) \psi_{\alpha}^{*}(y) A_{\alpha}(y) \nabla\left(y_{i}+\xi_{i, \alpha}(y)\right) \cdot \nabla\left(y_{j}+\xi_{j, \alpha}(y)\right) \mathrm{d} y \\
& +\sum_{\alpha, \beta=1}^{K} \frac{1}{2} \int_{Y} \psi_{\beta}(y) \psi_{\alpha}^{*}(y)\left(\mu_{\infty} \sigma_{\alpha, \beta}(y)-\Sigma_{\alpha, \beta}(y)\right) \\
& +\sum_{\alpha, \beta=1}^{K} J_{i} \int_{Y} \sigma_{\alpha, \beta}(y) \psi_{\beta} \psi_{\alpha}^{*}\left(\xi_{j, \beta}(y)-\xi_{j, \alpha}\right) \\
& +\sum_{\alpha, \beta=1}^{K} J_{j} \int_{Y} \sigma_{\alpha, \beta}(y) \psi_{\beta} \psi_{\alpha}^{*}\left(\xi_{i, \beta}(y)-\xi_{i, \alpha}\right)
\end{aligned}
$$

where the auxiliary functions $\left(\xi_{i}\right)_{1 \leqslant i \leqslant N}$ are uniquely defined up to a constant multiple of $\mathbb{1}=(1, \ldots, 1) \in \mathbb{R}^{K}$ by system $(3.20)$, for $\theta=0$. For this very value of $\theta$, after the following change of variable,

$$
\zeta_{i, \alpha}=\xi_{i, \alpha} \psi_{\alpha}, \quad 1 \leqslant \alpha \leqslant K
$$

system (3.20) becomes system (3.4) with a different source term,

$$
Z_{i, \alpha}=\frac{1}{\psi_{\alpha}(y)} \operatorname{div}\left(A_{\alpha}(y) \psi_{\alpha}^{2} \nabla y_{i}\right)+J_{i} \sum_{\beta=1}^{K} \sigma_{\alpha, \beta}(y) \psi_{\beta}(y), \quad 1 \leqslant \alpha \leqslant K
$$

For all $1 \leqslant i \leqslant N$, system (3.4) is the cell eigenvalue problem (1.3), with the additional source term $Z_{i}$. We can check that a solution exists since the Fredholm alternative holds, that is, $Z_{i}$ is orthogonal to $\psi^{*}$,

$$
\begin{aligned}
\sum_{\alpha=1}^{K} \int_{Y} Z_{i, \alpha}(y) \cdot \psi_{\alpha}^{*}(y)=- & \int_{Y} A_{\alpha} \psi_{\alpha}^{2} \nabla y_{i} \cdot \nabla\left(\frac{\psi_{\alpha}^{*}}{\psi_{\alpha}}\right) \mathrm{d} y \\
& +J_{i} \sum_{\alpha, \beta=1}^{K} \int_{Y} \sigma_{\alpha, \beta}(y) \psi_{\beta}(y) \cdot \psi_{\alpha}^{*}(y) \mathrm{d} y \\
=- & J_{i}+J_{i} \times 1=0
\end{aligned}
$$

Therefore, with only a small change in (3.4), i.e. adding the extra source term $Z_{i}$, we obtain, with the same computational scheme as in the drift-free case, a second-order expansion of $\lambda(\theta)$ near $\theta=0$, which is

$$
\lambda(\theta)=\mu_{\infty}+J \cdot \theta-\bar{D} \theta \cdot \theta+O\left(\theta^{3}\right)
$$


where each term $J$ and $\bar{D}$ are computed for $\theta=0$. Note that we also have a first-order development of the eigenvector $\psi_{\theta}$ in $\theta=0$, which is

$$
\psi_{\theta, \alpha}=\psi_{\alpha}\left(1+\sum_{i=1}^{N} \theta_{i} \cdot \xi_{i, \alpha}\right)+O\left(\theta^{2}\right)
$$

The major inconvenience of the above expansions (5.6) and (5.7) is that they are expressed in terms of $\theta$, i.e. suppose that $\theta$ is small and we do not know what the value of $\theta_{\infty}$ is. We shall now show that if $J$ is small, similar expansions be can obtained in terms of $J$ given (5.1), which only depend on the periodic direct and adjoint eigenvectors $\psi$ and $\psi^{*}$.

Proposition 5.1. Suppose that $J$ given by (5.1) is small. Then the maximum eigenvalue $\lambda_{\infty}$ is given by

$$
\lambda_{\infty}=\mu^{\infty}+\frac{1}{2}\left(\bar{D}_{S}\right)^{-1} J \cdot J+O\left(|J|^{3}\right)
$$

where $\mu^{\infty}$ is the first eigenvalue of the periodic cell problem (1.3). Furthermore, the drift factor $\theta_{\infty}$ is given by

$$
\theta_{\infty}=\frac{1}{2}\left(\bar{D}_{S}\right)^{-1} J+O\left(|J|^{2}\right)
$$

where the positive-definite matrix $\bar{D}_{S}$ is given by its entries

$$
\begin{aligned}
\left(\bar{D}_{S}\right)_{i, j}=\sum_{\alpha=1}^{K} \int_{Y} \psi_{\alpha}(y) \psi_{\alpha}^{*}(y) A_{\alpha}(y) \nabla\left(y_{i}+\xi_{i, \alpha}(y)\right) \cdot \nabla\left(y_{j}+\xi_{j, \alpha}(y)\right) \mathrm{d} y \\
+\sum_{\alpha, \beta=1}^{K} \frac{1}{2} \int_{Y} \psi_{\beta}(y) \psi_{\alpha}^{*}(y)\left(\mu_{\infty} \sigma_{\alpha, \beta}(y)-\Sigma_{\alpha, \beta}(y)\right) \\
\quad \times\left(\xi_{i, \alpha}(y)-\xi_{i, \beta}(y)\right) \cdot\left(\xi_{j, \alpha}(y)-\xi_{j, \beta}(y)\right) \mathrm{d} y
\end{aligned}
$$

and functions $\left(\xi_{i, \alpha}\right)_{1 \leqslant i \leqslant N, 1 \leqslant \alpha \leqslant K}$ are given, after the change of variable $\zeta_{i, \alpha}=$ $\xi_{i, \alpha} \psi_{\alpha}$, by

$$
\left.\begin{array}{c}
-\operatorname{div}\left(A(y) \nabla \zeta_{i}\right)+\Sigma(y) \zeta_{i}=\mu^{\infty} \sigma(y) \zeta_{i}+Z_{i} \quad \text { in } Y, \\
y \rightarrow \zeta_{i}(y) \quad Y \text {-periodic, }
\end{array}\right\}
$$

where $Z_{i}$ is given by (5.5). Finally, the homogenized matrix is given by

$$
\bar{D}_{\infty}=\bar{D}_{S}+O(|J|) \text {. }
$$

REMARK 5.2. In proposition 5.1, everything $\left(\mu^{\infty}, J, \bar{D}_{S}\right)$ is computed for $\theta=0$. This result can be compared with the one obtained by Larsen and Williams [17] for a one-dimensional example for the neutron transport equation.

It can be observed that $\bar{D}_{S}$ and the homogenized matrix $\bar{D}$ for the drift-free case (3.3) are very much alike. In fact, they would be equal if $J=0$. Note also that $\bar{D}_{S}$ would be the homogenized matrix if the computation had been made at $\theta=\theta_{\infty}$ instead of $\theta=0$. 
There exists an exact formula for $\theta_{\infty}$ in one dimension for the following eigenvalue problem with drift (see [7]),

$$
\begin{gathered}
\left.-\operatorname{div}\left(a\left(\frac{x}{\epsilon}\right) \nabla u_{\epsilon}\right)+\frac{b}{\epsilon} u_{\epsilon}(x)=\lambda_{\epsilon} u_{\epsilon}(x) \text { in }\right] m, n[ \\
u_{\epsilon}(m)=u_{\epsilon}(n)=0
\end{gathered}
$$

where $a$ is a 1-periodic positive function and $b$ is a constant. In such a case, the formula is simply

$$
\theta_{\infty}=\frac{b}{2 \int_{0}^{1} a(y) \mathrm{d} y}
$$

Let us first show that the drift factor $\theta_{\infty}$ is controlled by the modulus of $J$.

LEMMA 5.3. There exists a constant $C>0$, depending only on the coercivity constant of $A$ given by (2.2) and of the bounds of the direct and adjoint periodic eigenvectors $\psi$ defined in (1.3) and $\psi^{*}$ defined in (2.7), such that

$$
\left|\theta_{\infty}\right| \leqslant \frac{|J|}{C}
$$

Proof. From lemmas 4.1 and 4.3 , we know that the set

$$
\left\{\theta \in U \text { such that } \lambda(\theta) \geqslant \mu^{\infty}\right\}
$$

can be written $M^{-1}\left(\mathbb{R}_{+}\right)$, where $M$ is in $C^{\infty}\left(\mathbb{R}^{N}, \mathbb{R}\right)$, satisfies $\left(\partial M / \partial \theta_{i}\right)(0)=J_{i}$, $M(0)=0$ and

$$
\forall \zeta \in \mathbb{R}^{N}, \quad-\sum_{i, j=1}^{N} \frac{\partial^{2} M}{\partial \theta_{i} \partial \theta_{j}}(\theta) \zeta_{i} \zeta_{j} \geqslant C \sum_{i=1}^{N}\left|\zeta_{i}\right|^{2} \quad \text { for all } \theta \in \mathbb{R}^{N},
$$

where $C$ depends on the smallest of the coercivity constants of the diffusion operators $\left(A_{\alpha, i, j} \psi_{\alpha} \psi_{\alpha}^{*}\right)_{1 \leqslant i, j \leqslant N}$, on $\Sigma$ and $\mu^{\infty}$, but does not depend on $\theta$. The conclusion follows.

Proof of proposition 5.1. If we decompose each component of $\psi_{\infty}$ and $\psi_{\infty}^{*}$ in an exponential times periodic form, that is,

$$
\psi_{\infty}=\exp \left(\theta_{\infty} \cdot y\right) \varphi_{\infty}(y), \quad \psi_{\infty}^{*}=\exp \left(-\theta_{\infty} \cdot y\right) \varphi_{\infty}^{*}(y),
$$

we obtain that $(2.12)$, which characterizes $\theta_{\infty}$, transforms into

$$
\begin{aligned}
& -2 \sum_{\alpha=1}^{K} \int_{Y} \varphi_{\infty, \alpha}(y) \varphi_{\infty, \alpha}^{*}(y) A_{\alpha}(y) \theta_{\infty} \cdot e_{i} \\
& \quad+\sum_{\alpha=1}^{K} \int_{Y} A_{\alpha}(y)\left(\varphi_{\infty, \alpha}(y) \nabla \varphi_{\infty, \alpha}^{*}(y)-\varphi_{\infty, \alpha}^{*}(y) \nabla \varphi_{\infty, \alpha}(y)\right) \cdot e_{i}=0 .
\end{aligned}
$$

If we define the constant matrix $\tilde{A}_{\infty, i, j}$ by its entries

$$
\tilde{A}_{\infty, i, j}=\sum_{\alpha=1}^{K} \int_{Y} \varphi_{\infty, \alpha}(y) \varphi_{\infty, \alpha}^{*}(y) A_{\alpha, i, j}(y)
$$


and the constant vector $J_{\infty}$ by its components

$$
J_{\infty, i}^{\#}=\sum_{\alpha=1}^{K} \int_{Y} A_{\alpha}(y)\left(\varphi_{\infty, \alpha}(y) \nabla \varphi_{\infty, \alpha}^{*}(y)-\varphi_{\infty, \alpha}^{*}(y) \nabla \varphi_{\infty, \alpha}(y)\right) \cdot e_{i},
$$

we obtain that identity (5.13) writes as follows:

$$
2 \tilde{A}_{\infty, i, j} \theta_{\infty}-J_{\infty}^{\#}=0 .
$$

A first-order development of each term of formula (5.14) in $\theta_{\infty}$ gives

$$
2 \bar{D}_{S} \theta_{\infty}=J+O\left(\left|\theta_{\infty}\right|^{2}+|J|\left|\theta_{\infty}\right|\right) .
$$

and since we have obtained in lemma 5.3 that $\theta_{\infty}$ is controlled by $J$, equation $(5.15)$ is also

$$
2 \bar{D}_{S} \theta_{\infty}=J+O\left(|J|^{2}\right)
$$

Formula (4.19) shows that $\bar{D}_{S}$ is positive definite, thus we have obtained (5.9). On the other hand, the formula of the Hessian matrix $\bar{D}(5.3)$ in $\theta=0$ shows that it writes as follows:

$$
\bar{D}=\bar{D}_{S}+J \otimes X+X \otimes J
$$

Therefore, formula (5.6) can be simplified as follows:

$$
\lambda(\theta)=\mu^{\infty}+J \cdot \theta-\bar{D}_{S} \theta \cdot \theta+O\left(|J||\theta|^{2}\right) .
$$

Using (5.9) and (5.16), we obtain formula (5.8). Finally, notice that

$$
\begin{aligned}
\bar{D}_{\infty} & =-\frac{1}{2} \frac{\partial^{2} \lambda}{\partial \theta_{i} \partial \theta_{j}}\left(\theta_{\infty}\right) \\
& =-\frac{1}{2} \frac{\partial^{2} \lambda}{\partial \theta_{i} \partial \theta_{j}}(0)+O\left(\left|\theta_{\infty}\right|\right) \\
& =\bar{D}_{S}+O\left(|J|+\left|\theta_{\infty}\right|\right) \\
& =\bar{D}_{S}+O(|J|) .
\end{aligned}
$$

We shall now use the approximation obtained for $\theta_{\infty}$ to derive a first-order approximate formula for the homogenized diffusion operator. In the following, we shall note $\theta_{\mathrm{a}}$ the approximation of $\theta_{\infty}$, i.e.

$$
\theta_{\mathrm{a}}=\frac{1}{2}\left(\bar{D}_{S}\right)^{-1} J
$$

A striking property of the application $\theta \rightarrow \lambda(\theta)$ is that its Hessian matrix

$$
\frac{\partial^{2} \lambda}{\partial \theta_{i} \partial \theta_{j}}\left(\theta_{\infty}\right)
$$

is equal to $-2 \bar{D}_{\infty}$, the homogenized matrix. Since $\theta \rightarrow \lambda(\theta)$ is smooth, the computation of $\left(\partial^{2} \lambda / \partial \theta_{i} \partial \theta_{j}\right)\left(\theta_{\mathrm{a}}\right)$ will provide a first-order approximation of the homogenized 
matrix $\bar{D}_{\infty}$. Let $\left(\psi_{\mathrm{a}}, \psi_{\mathrm{a}}^{*}, \lambda_{\mathrm{a}}\right)$ be the first eigen-triplet defined by (1.4) and (2.8) at the point $\theta=\theta_{\mathrm{a}}$, i.e.

$$
\left.\begin{array}{c}
-\operatorname{div}\left(A(y) \nabla \psi_{\mathrm{a}}\right)+\Sigma(y) \psi_{\mathrm{a}}=\lambda_{\mathrm{a}} \sigma(y) \psi_{\mathrm{a}} \\
y \rightarrow \psi_{\mathrm{a}}(y) \mathrm{e}^{-\theta_{\mathrm{a}} \cdot y} \quad Y \text {-periodic }
\end{array}\right\}
$$

and

$$
\left.\begin{array}{c}
-\operatorname{div}\left(A(y) \nabla \psi_{\mathrm{a}}^{*}\right)+\Sigma^{\mathrm{T}}(y) \psi_{\mathrm{a}}^{*}=\lambda_{\mathrm{a}} \sigma^{\mathrm{T}}(y) \psi_{\mathrm{a}}^{*}, \\
y \rightarrow \psi_{\mathrm{a}}^{*}(y) \mathrm{e}^{\theta_{\mathrm{a}} \cdot y} \quad Y \text {-periodic, }
\end{array}\right\}
$$

normalized in the following way:

$$
\sum_{\alpha=1}^{K} \int_{Y} \psi_{a, \alpha}(y) \cdot \psi_{a, \alpha}(y) \mathrm{d} y=1 \quad \text { and } \quad \sum_{\alpha, \beta=1}^{K} \int_{Y} \sigma_{\alpha, \beta}(y) \psi_{a, \beta}(y) \cdot \psi_{a, \alpha}^{*}(y) \mathrm{d} y=1 .
$$

A formula similar to (5.3) can be derived, and we obtain

$$
\begin{aligned}
-\frac{1}{2} \frac{\partial^{2} \lambda}{\partial \theta_{i} \partial \theta_{j}}\left(\theta_{\mathrm{a}}\right)=\sum_{\alpha=1}^{K} & \int_{Y} \psi_{a, \alpha}(y) \psi_{a, \alpha}^{*}(y) A_{\alpha}(y) \nabla\left(y_{i}+\xi_{i, \alpha}^{a}(y)\right) \cdot \nabla\left(y_{j}+\xi_{j, \alpha}^{a}(y)\right) \mathrm{d} y \\
& +\sum_{\alpha, \beta=1}^{K} \frac{1}{2} \int_{Y} \psi_{a, \beta}(y) \psi_{a, \alpha}^{*}(y)\left(\lambda_{\mathrm{a}} \sigma_{\alpha, \beta}(y)-\Sigma_{\alpha, \beta}(y)\right) \\
& \times\left(\xi_{i, \alpha}^{a}(y)-\xi_{i, \beta}^{a}(y)\right) \cdot\left(\xi_{j, \alpha}^{a}(y)-\xi_{j, \beta}^{a}(y)\right) \mathrm{d} y \\
& +\sum_{\alpha, \beta=1}^{K} J_{i}^{a} \int_{Y} \sigma_{\alpha, \beta}(y) \psi_{a, \beta} \psi_{a, \alpha}^{*}\left(\xi_{j, \beta}^{a}(y)-\xi_{j, \alpha}^{a}\right) \\
& +\sum_{\alpha, \beta=1}^{K} J_{j}^{a} \int_{Y} \sigma_{\alpha, \beta}(y) \psi_{a, \beta} \psi_{a, \alpha}^{*}\left(\xi_{i, \beta}^{a}(y)-\xi_{i, \alpha}^{a}\right),
\end{aligned}
$$

where, for all $\left(\xi_{i}^{a}\right)_{1 \leqslant i \leqslant N}$, the functions are uniquely defined up to a constant multiple of $\mathbb{1}=(1, \ldots, 1) \in \mathbb{R}^{K}$ by

$$
\zeta_{i, \alpha}^{a}=\xi_{i, \alpha}^{a} \psi_{\alpha}^{a}, \quad 1 \leqslant \alpha \leqslant K,
$$

and $\zeta_{i, \alpha}^{a}$ is solution of

$$
\left.\begin{array}{c}
-\operatorname{div}\left(A(y) \nabla \zeta_{i}^{a}\right)+\Sigma(y) \zeta_{i}^{a}=\lambda_{\mathrm{a}} \sigma(y) \zeta_{\mathrm{a}}^{i}+Z_{i}^{a}, \\
y \rightarrow \psi_{\mathrm{a}}(y) \mathrm{e}^{-\theta_{\mathrm{a}} \cdot y} \quad Y \text {-periodic }
\end{array}\right\}
$$

with

$$
Z_{i, \alpha}=\frac{1}{\psi_{a, \alpha}(y)} \operatorname{div}\left(A_{\alpha}(y) \psi_{a, \alpha}^{2} \nabla y_{i}\right)+J_{i, a} \sum_{\beta=1}^{K} \sigma_{\alpha, \beta}(y) \psi_{a, \beta}(y), \quad 1 \leqslant \alpha \leqslant K .
$$

\section{Acknowledgments}

This work as been completed as a part of a $\mathrm{PhD}$ thesis granted by the Commissariat à l'Energie Atomique, France. The author gratefully thanks his advisor, Grégoire 
Allaire, for his constant help and support. The existence and regularity results presented in $\S 2$ are the result of fruitful discussions with Guido Sweers, for which the author also thanks him. Finally, thanks are also due to the anonymous referee for his detailed comments and suggestions.

\section{References}

1 G. Allaire and Y. Capdeboscq. Homogenization of a spectral problem in neutronic multigroup diffusion. Comput. Meth. Appl. Mech. Engng 187 (2000), 91-117.

2 G. Allaire and F. Malige. Analyse asymptotique spectrale d'un problème de diffusion neutronique. C. R. Acad. Sci. Paris Sér. I 324 (1997), 939-944.

3 H. Amann. Fixed point equations and nonlinear eigenvalue problems in ordered Banach spaces. SIAM Rev. 18 (1976), 620-710.

4 P. Anselone. Collectively compact operator approximation theory and applications to integral equations (Englewood Cliffs, NJ: Prentice-Hall, 1971).

5 G. Bal. Homogenization of the neutron transport criticality spectral equation with drift. ESAIM Control Optim. Calc. Var. 6 (2001), 613-627.

6 A. Bensoussan, J.-L. Lions and G. Papanicolaou. Asymptotic analysis for periodic structures (Amsterdam: North-Holland, 1978).

7 Y. Capdeboscq. Homogenization of a diffusion equation with drift. C. R. Acad. Sci. Paris Sér. I 327 (1998), 807-812.

8 F. Chatelin. Spectral approximation of linear operators. In Computer science and applied mathematics (Academic, 1983).

9 V. Deniz. The theory of neutron leakage in reactor lattices. In Handbook of nuclear reactor calculations (ed. Y. Ronen), vol. II, pp. 409-508 (Boca Raton, FL: Chemical Rubber Company, 1986).

10 D. Gilbarg and N. Trudinger. Elliptic partial differential equations of second order. In Comprehensive studies in mathematics, 2nd edn (Springer, 1983).

11 G. Habetler and M. Martino. The multigroup diffusion equations of reactor physics. Technical Report KAPL 1886, Knolls Atomic Power Laboratory (1958).

T. Kato. Perturbation theory for linear operators, 2nd edn (Springer, 1984).

13 S. Kozlov and A. L. Pyatnitskii. Degeneration of effective diffusion in the presence of periodic potential. Annls Inst. H. Poincaré Analyse Non Linéaire 32 (1996), 571-587.

14 M. Krein and M. Rutman. Linear operators leaving invariant a cone in a Banach space. In Functional analysis and measure theory, vol. 10, pp. 199-325 (Providence, PA: American Mathematical Society, 1962).

15 E. W. Larsen. Neutron transport and diffusion in inhomogeneous media. J. Math. Phys. 16 (1975), 1421-1427.

16 E. W. Larsen. Neutron transport and diffusion in inhomogeneous media. Nucl. Sci. Engng 60 (1976), 357-368.

17 E. W. Larsen and M. Williams. Neutron drift in heterogenous media. Nucl. Sci. Engng 65 (1978), 290-302.

18 F. Malige. Etude mathématique et numérique de l'homogénéisation des assemblages combustibles d'un cœur de réacteur nucléaire. Thèse de Doctorat de l' école Polytechnique (1996).

19 E. Mitidieri and G. Sweers. Weakly coupled elliptic systems and positivity. Math. Nachr. 173 (1995), 259-286.

20 A. L. Piatnitski. Homogenization of singularly perturbed operators. In Homogenization and applications to material sciences (ed. D. Cioranescu et al.). GAKUTO International Series of Mathematics and Science Applications, vol. 9, pp. 355-361 (Tokyo: Gakkōtosho, 1995).

21 A. L. Piatnitski. Asymptotic bevahiour of the ground state of singularly perturbed elliptic equations. Commun. Math. Phys. 197 (1998), 527-551.

22 J. Planchard. Méthodes mathématiques en neutronique. Collection de la Direction des Etudes et Recherches d'EDF (Paris: Eyrolles, 1995).

23 M. Reed and B. Simon. Methods of modern mathematical physics. IV. Analysis of operators (Academic, 1978). 
24 R. Sentis. Approximation and homogenization of a transport process. SIAM J. Appl. Math. 39 (1980), 134-141.

25 G. Stampacchia. Le problème de Dirichlet pour les équations élliptiques du second ordre à coefficients discontinus. Annls Inst. Fourier 15 (1965), 189-258.

26 G. Sweers. Strong positivity in $c(\bar{\Omega})$ for elliptic systems. Math. Z. 209 (1992), 251-272.

(Issued 21 June 2002) 